\title{
Loss of NEDD4 contributes to RTP801 elevation and neuron toxicity: implications for Parkinson's disease
}

\author{
Mercè Canal ${ }^{1,5}$, Núria Martín-Flores ${ }^{1,5}$, Leticia Pérez-Sisqués ${ }^{1,5}$, Joan Romaní- \\ Aumedes ${ }^{1,5}$, Bekir Altas ${ }^{2}$, Heng-Ye Man ${ }^{3}$, Hiroshi Kawabe ${ }^{2}$, Jordi Alberch ${ }^{1,4,5}$ and \\ Cristina Malagelada ${ }^{1,5}$ \\ ${ }^{1}$ Department of Biomedicine, Faculty of Medicine, Universitat de Barcelona, Barcelona, Catalonia, Spain \\ 2 Department of Molecular Neurobiology, Max Planck Institute of Experimental Medicine, Göttingen, Germany \\ ${ }^{3}$ Department of Biology, Pharmacology and Experimental Therapeutics, Boston University, Boston, Massachusetts, United \\ States of America \\ ${ }^{4}$ IDIBAPS-Institut d'Investigacions Biomèdiques August Pi i Sunyer, Centro de Investigación Biomédica en Red sobre \\ Enfermedades Neurodegenerativas, Barcelona, Catalonia, Spain \\ ${ }^{5}$ Institut de Neurociències, Universitat de Barcelona, Barcelona, Catalonia, Spain \\ Correspondence to: Cristina Malagelada, email: cristina.malagelada@ub.edu
}

Keywords: Parkinson's disease, neurodegeneration, lysosome, NEDD4, ubiquitin, Pathology Section

Received: May 23, 2016

Accepted: July 22, 2016

Published: August 02, 2016

\section{ABSTRACT}

Parkinson's disease (PD) is a disorder characterized by the degeneration of certain neuronal populations in the central and peripheral nervous system. One of the hallmarks of the disease is the toxic accumulation of proteins within susceptible neurons due to major impairment in the $d$ egradation/clearance protein systems.

RTP801 is a pro-apoptotic protein that is sufficient and necessary to induce neuronal death in cellular and animal models of PD. RTP801 is also upregulated in sporadic and parkin mutant PD brains. Here, we report the role of NEDD4, an E3 ligase involved in a-synuclein degradation and PD pathogenesis, in the regulation of RTP801 protein levels and toxicity. NEDD4 polyubiquitinates RTP801 in a cell-free system and in cellular cultures, and they interact physically. NEDD4 conjugates K63-ubiquitin chains to RTP801 and targets it for degradation. NEDD4 regulates RTP801 protein levels in both cultured cells and in the brain tissue. NEDD4 levels are diminished in nigral neurons from human PD brains. Interestingly, neurotoxin 6-OHDA decreases dramatically NEDD4 protein expression but elevates RTP801 protein levels. Moreover, NEDD4 protects neuronal PC12 cells from both 6-OHDA and RTP801-induced toxicity. In primary cortical neurons, NEDD4 knockdown toxicity is mediated by RTP801 since the double knockdown of RTP801 and NEDD4 abrogates the loss of phospho Ser473Akt and the appearance of caspase-cleaved spectrin fragments.

Thus, NEDD4 ligase regulates RTP801 and is sensitive to PD-associated oxidative stress. This suggests that NEDD4 loss of function in PD could contribute importantly into neuronal death by elevating RTP801.

\section{INTRODUCTION}

In Parkinson's disease (PD), the mechanisms by which specific subpopulations of both central and peripheral neurons degenerate are not yet elucidated [1]. Moreover, only palliative treatments exist to ameliorate the clinical manifestations of the disease but they do not prevent neuron degeneration and death [2-4].
RTP801 is a stress-regulated protein with several functions depending on the cellular context [5]. In a variety of neuronal systems, RTP801 overexpression is sufficient to trigger cell death $[5,6]$. In cellular models of PD, shRNA-mediated RTP801 knockdown is protective toward cell death induced by 6-hydroxydopamine (6OHDA) or MPP+ [6]. Consistently, RTP801 is required for cell death triggered by MPP+/MPTP in in vivo model of PD [6]. 
RTP801 triggers neuron cell death by a sequential mechanism in which it first inactivates mechanistic target of Rapamycin (mTOR) and then, as a consequence, inhibits the neuronal survival kinase Akt, which is also a substrate of mTOR [6, 7]. In human postmortem tissue, RTP801 was found to be highly upregulated in neuromelanin (NM) positive neurons in the SNpc of both sporadic [6] and parkin mutant PD patients [8] in comparison with control non-PD brains. Also, in accordance with the mechanism proposed from our in vitro studies, very low levels of phospho-Akt (both Serine 473 and Threonine 308) were observed in nigral PD neurons in comparison to non-PD brains [7].

One remarkable feature of RTP801 protein is its very short half-life (2-5 $\mathrm{min})[9,10]$, suggesting that its synthesis and degradation are regulated strictly and dynamically. Our previous study demonstrated that parkin, a RING E3 ligase, ubiquitinates RTP801 and targets it for ubiquitin proteasome system (UPS) [8].

Neural precursor cell expressed, developmentally down-regulated 4 (NEDD4) is one of the most abundant ubiquitin E3 ligases in mammalian neurons [11]. NEDD4 ubiquitinates proteins, targeting them for proteasomal or lysosomal degradation [12]. In developing neurons, NEDD4 plays crucial roles in axon growth and dendrite sprouting $[13,14]$. In a context of PD, NEDD4 protects neurons from alpha synuclein toxicity by ubiquitinating it and mediating its lysosomal degradation [15, 16]. Interestingly, NEDD4 staining is very strong in nigral neurons containing Lewy bodies (LB) in the human Substantia Nigra (SN) and the Locus Coeruleus (LC) from patients with LB pathologies [15]. Furthermore, NEDD4 presents a single nucleotide polymorphism (SNP) that has been associated with a major risk factor for sporadic PD in a whole genome association study (GWAS) [17].

Here, we identify NEDD4 as a novel E3 ubiquitin ligase for RTP801, controlling its homeostasis. Importantly, NEDD4 is downregulated in remaining nigral neurons from PD brains. Moreover, 6-OHDA downregulates NEDD4 in neural cultures and NEDD4 deregulation contributes to toxic elevation of RTP801 in cellular models of PD.

\section{RESULTS}

\section{RTP801 is degraded by the lysosomal pathway and polyubiquitinated by NEDD4}

In our previous work we showed that RTP801 has a very short half-life and is mostly degraded by the proteasome [8-10]. Hence, we first asked whether lysosomal pathway could contribute to RTP801 protein degradation. As cellular models we used NGFdifferentiated PC12 cells, a cell line that resembles sympathetic neuroblasts which is a neuronal population also affected in PD [3, 18], and rat primary cortical neurons, that are also sensitive to 6-OHDA [19] or alphasynuclein toxicity [20]. We first exposed the cultures to chloroquine, a lysosomotropic agent that prevents endosomal acidification and thus inhibits lysosomal fusion and protein degradation [21, 22]. Sister cultures were treated with proteasome inhibitors epoxomicin or MG132. Western immunoblotting (WB) showed that RTP801 was accumulated upon the inhibition of the proteasome, as previously described [8]. Interestingly, chloroquine induced a significant elevation of RTP801 after 4-hour exposure in both cultured cell types (Figure 1a).

Given that ubiquitination is involved in lysosomal degradation [23], we next investigated which E3 ligase could mediate lysosomal degradation of RTP801. We investigated NEDD4, which is an E3 ligase involved in $\mathrm{PD}$ by regulating alpha-synuclein proteostasis. Hence, we assessed whether recombinant NEDD4 polyubiquitinated RTP801 in a cell free assay. For this purpose, we incubated recombinant NEDD4, recombinant GST-RTP801, biotinylated Ubiquitin, an E1 ubiquitin-activating enzyme, and $\mathrm{UbcH} 5 \mathrm{~b}$ at $37^{\circ} \mathrm{C}$ for $90 \mathrm{~min}$. After incubation, RTP801 was immunoprecipitated and analyzed by WB. By using an anti-biotin antibody, we observed the appearance of high molecular weight ubiquitinated RTP801 (HMW-Ub RTP801) species when all the enzymes and the substrates were present in the reaction (Figure 1b). Hence, NEDD4 is able to directly ubiquitinate RTP801 in a cell-free system.

To explore whether this process takes place in a cellular model, wild type (WT) NEDD4, RTP801, and Hemagglutinin (HA)-tagged ubiquitin (HA-Ub) were overexpressed in HEK293 cells. Two days later, cells were harvested and RTP801 was immunoprecipitated. WB analysis of the immunocomplexes showed that ectopic NEDD4 significantly increased the appearance of HMWUb RTP801 with different lengths of polyubiquitin chains (Figure 1c). These results suggest that NEDD4 enhances polyubiquitination of RTP801 in cellular cultures.

To investigate which kind of ubiquitin chains NEDD4 uses preferentially with RTP801, we transfected HEK293 cells with expression vectors for pCMS-eGFPRTP801, NEDD4, and modified HA-Ubiquitin. The ubiquitin plasmids have all the lysine residues mutated to arginine except for $48^{\text {th }}$ or $63^{\text {rd }}$ lysine residue (K48 or $\mathrm{K} 63$ ). This preferentially leads to the formation of K48or K63-linked polyubiquitin chains at the substrates. WB analysis of RTP801 immunoprecipitates showed that the RTP801 HMW smear was increased only in the presence of overexpressed NEDD4 along with HA-Ub-K63. Thus, NEDD4 polyubiquitinates RTP801 preferentially with K63-linked polyubiquitin chains (Figure 1d). Note that with HA-Ub-K48, the appearance of RTP801 HMW smear is independent of ectopic NEDD4. This indicates that RTP801 is ubiquitinated with K48 chains by other ligases, like parkin, and degraded by the proteasome [8]. 


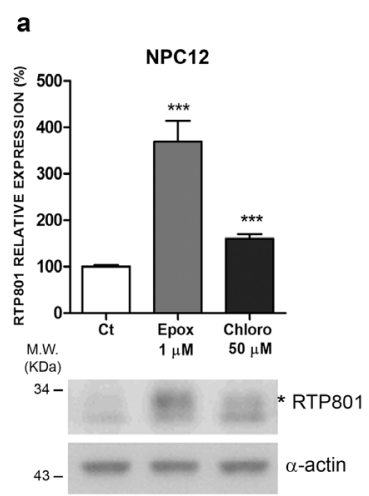

c
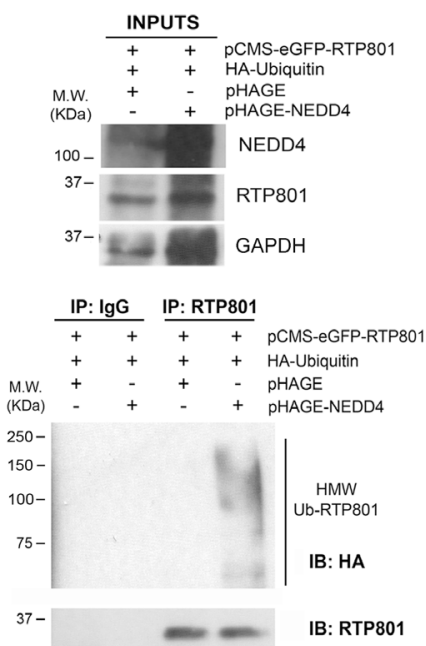

b

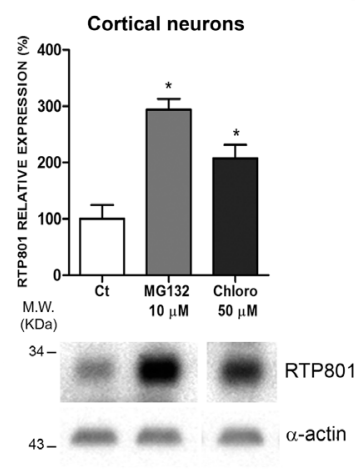

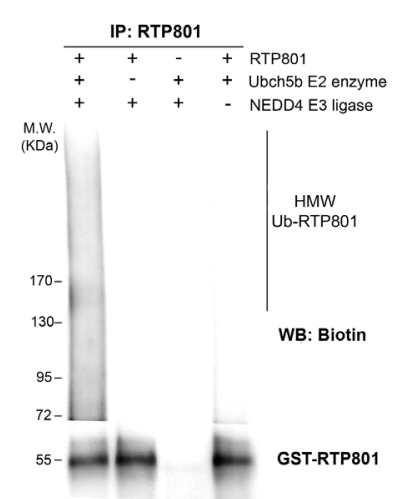

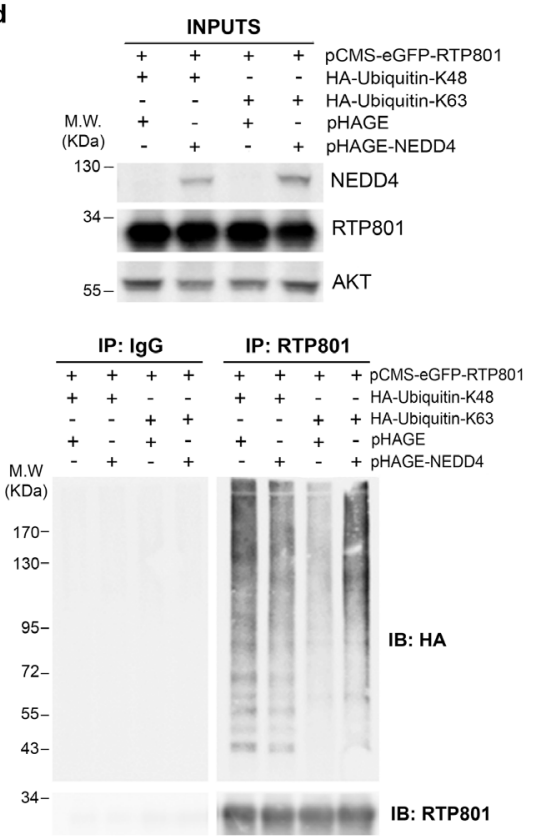

Figure 1: RTP801 is polyubiquitinated by NEDD4 and degraded by the lysosomal pathway. a. RTP801 can be degraded by both the ubiquitin proteasome system and by the lysosomal pathway. NGF-differentiated PC12 cells or cortical neurons were treated during 4 hours with $1 \mu \mathrm{M}$ epoxomycin, $10 \mu \mathrm{M}$ MG132 or $50 \mu \mathrm{M}$ chloroquine, and cell lysates were subjected to Western Blot. Membranes were probed first for RTP801 and then with $\alpha$-actin, as a loading control. All samples were immunoblotted in the same membrane, but one irrelevant lane was deleted in the second panel. Graphs represent densitometric values (mean \pm SEM) normalized to $\alpha$-actin of three independent experiments in triplicates. Student's $t$-test, $* * * P<0.001$ and $* P<0.05$ versus controls. b. NEDD4 polyubiquitinates RTP801 in a cell free assay. Recombinant NEDD4 E3 ligase, recombinant GST-RTP801, UbcH5b E2 enzyme, E1 enzyme, biotinylated ubiquitin and ATP were mixed and incubated at $37^{\circ} \mathrm{C}$ for $90 \mathrm{~min}$. RTP801 was immunoprecipitated and immunocomplexes were analyzed by Western Blot. The membrane was incubated with Avidin/Biotin and then with chemiluminiscent peroxidase substrate solution (upper panel) and reprobed for RTP801 (lower panel). A representative image of three independent experiments is shown. (HMW Ub-RTP801, high molecular weight ubiquitinated RTP801) c. NEDD4 enhances RTP801 polyubiquitination. HEK293 cells were transfected with pHAGE or pHAGE-NEDD4 along with HA-ubiquitin and pCMS-eGFP-RTP801 constructs. Forty-eight hours post-transfection either RTP801 was immunoprecipitated or non-specific rabbit immunoglobulins ( $\mathrm{Rb} \mathrm{IgG}$ ) were added. Whole cell lysates (inputs) and the immunocomplexes were analyzed by Western Blot with anti-HA, anti-RTP801, anti-NEDD4 and anti-GAPDH (loading control) antibodies. A representative image of two independent experiments is shown. HMW Ub-RTP801 = High molecular weight ubiquitinated RTP801; IP = immunoprecipitation; IB = immunoblot. d. NEDD4 polyubiquitinates RTP801 with Ub-K63 chains. HEK293 cells were transfected with pCMS-eGFP-RTP801, along with pRK5-HA-Ub-K48 or pRK5-HA-Ub-K63 and pHAGE or pHAGE-NEDD4 as indicated. Forty-eight hours later, cultures were harvested and RTP801 was immunoprecipitated. Non-specific rabbit immunoglobulins (Rb IgG) were used as a negative control. Whole cell lysates (inputs) and RTP801 immunocomplexes were resolved in a Western Blot. Membrane was probed for HA, and reprobed for RTP801, for NEDD4 and for AKT as loading control. All samples were immunoblotted in the same membrane, but some irrelevant bands were deleted. Note the high molecular weight smears corresponding to polyubiquitinated RTP801. A representative image of three independent experiments is shown. IP = immunoprecipitation; IB = immunoblot. 
In conclusion, RTP801 can be subjected to lysosomal degradation and is conjugated with K63-linked polyubiquitin chains by NEDD4.

\section{NEDD4 and RTP801 interact in cellular models}

In order to investigate whether NEDD4 and RTP801 interact in living cells, we overexpressed both NEDD4 and RTP801 in HEK293 cells. Cultures were incubated for 2 hours with DSP, a crosslinker agent to preserve weak interactions between proteins, and then RTP801 was immunoprecipitated. WB analysis of the immunocomplexes showed that ectopic RTP801 pulled down overexpressed NEDD4 (Figure 2a).

In order to study the complex formation in nontransfected cells, we next investigated whether the endogenous NEDD4 and RTP801 interact in NGFdifferentiated PC12 cells. For this purpose, cultures were treated with $1 \mu \mathrm{M}$ epoxomycin for 2 hours to abrogate RTP801 fast degradation and then, they were exposed to the crosslinker DSP for two hours more. Then, endogenous RTP801 was immunoprecipitated. WB analysis showed that NEDD4 was able to interact with endogenous RTP801 (Figure 2b).

We next explored whether NEDD4 and RTP801 colocalize in cultured primary neurons. We overexpressed HA-tagged NEDD4 in primary rat cortical cultures and we detected ectopic NEDD4 with an antibody against the HA epitope. Interestingly, endogenous RTP801 co-localized with HA-tagged NEDD4 in the soma and in the neurites (Figure 2c) of transfected neurons.

Taken together, these experiments suggest the physical interaction of NEDD4 and RTP801 in several cellular models.

\section{NEDD4 regulates RTP801 protein levels in cellular models}

Our next question was whether NEDD4, by polyubiquitinating RTP801, could regulate RTP801 protein levels. In neuronal PC12 cells, we overexpressed WT NEDD4 and its inactive mutant, with the catalytic cysteine 867 mutated to a serine (NEDD4-C867S). After 48 hours, cell lysates were analyzed by WB. Ectopic NEDD4 diminished in a $25-30 \%$ the levels of endogenous RTP801 protein. Interestingly, the inactive mutant form of NEDD4 did not affect RTP801 levels (Figure 3a). We obtained the same results in rat primary cortical neurons (Figure 3b).

Still in cortical neurons, either ectopic NEDD4 or its mutant did not alter the messenger RNA levels of RTP801 (Figure 3c). These results indicate that NEDD4 does not regulate the transcription of RTP801.

We next asked whether NEDD4 knockdown elevates RTP801 protein levels. Therefore, in rat primary cortical neurons, NEDD4 expression was abrogated with specific shRNAs packed in lentiviruses. Six days later, cell lysates were analyzed by WB. Upon expression of shRNA for NEDD4, almost all NEDD4 protein was knocked down as shown in the top panel of Figure 3d. We observed a significant increase of RTP801 protein levels in the absence of NEDD4. In the same conditions, we also detected that the levels of phosphorylated residue S473 of the survival kinase Akt were diminished and the levels of caspase-cleaved spectrin fragment SBDP120, as readout of toxicity, were significantly elevated (Figure 3d). Thus, NEDD4 loss induces an elevation of RTP801 and is deleterious for cultured neurons.

Then, we assessed whether the NEDD4 deletion could affect the accumulation of RTP801 in an animal model. NEDD4 knockout mice are not viable after birth. For this reason we used NEDD4f/f;Emx ICre conditional knockout mice that only abolishes the expression of NEDD4 in glutamatergic neurons and glial cells in the cortex [13, 24, 25]. By WB, cortical lysates from 6-week old mice showed that the downregulation of NEDD4 was around 50\%. Accordingly, RTP801 levels were increased by a $25 \%$ in the conditional mice in comparison to the control NEDD4 $4^{\mathrm{f} / \mathrm{f}}$ littermates (Figure $3 \mathrm{e}$ ).

Together, these results show that NEDD4 regulates protein levels of RTP801 in cultured cortical neurons and in vivo.

\section{NEDD4 protects neuronal PC12 cells from ectopic RTP801 toxicity}

Overexpression of RTP801 induces around 50\% of cell death in cultured neurons and non-proliferating NGFdifferentiated PC12 cells $[6,8]$. With this rationale, we overexpressed pCMS-eGFP empty vector or with pCMSeGFP-RTP801 along with WT NEDD4 and the inactive mutant NEDD4-C867S. Twenty-four hours later, cell viability was assessed by scoring the number of living eGFP+ cells under epifluorescence microscopy. Only WT NEDD4 but not NEDD4-C867S protected cells from RTP801 toxicity (Figure 4a). Indeed, NEDD4 did not exert protection over non-ubiquitinable RTP801-KR mutant, with the 6 lysines mutated to arginines. These results support our notion that NEDD4 protects from RTP801 toxicity by ubiquitinating it (Figure $4 b$ ).

\section{PD neurotoxin 6-OHDA decreases NEDD4 and elevates RTP801 protein levels}

In our previous works we described that 6-OHDA elevated RTP801 protein levels transcriptionally and also posttranslationally, by interfering in its proteasomal degradation $[6,8,10]$. We observed that in culture, 6-OHDA was deleterious for parkin E3 ligase activity/ levels [8]. Based on this background, we next asked 

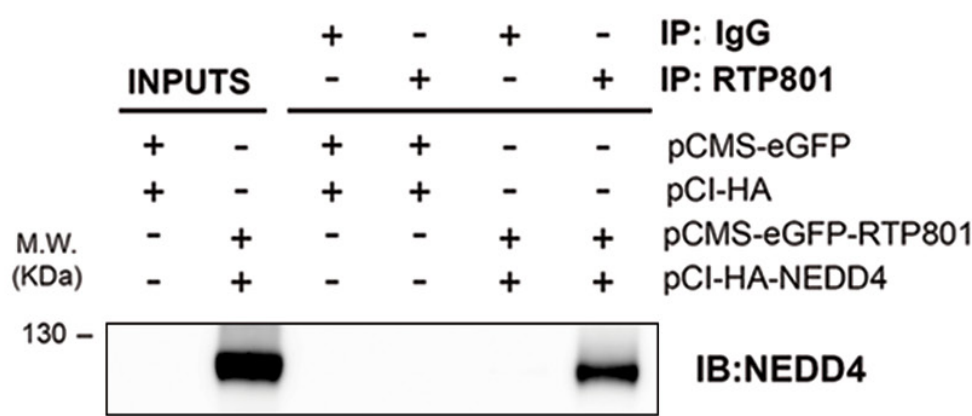

IB:NEDD4

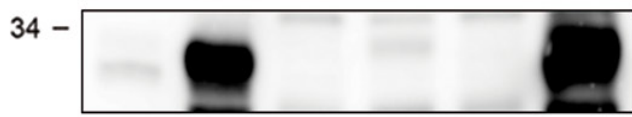

IB:RTP801

b

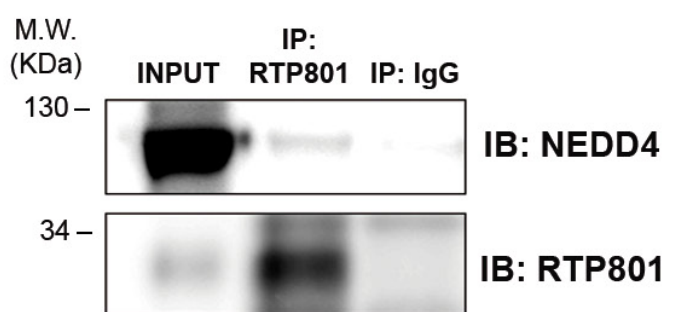

C

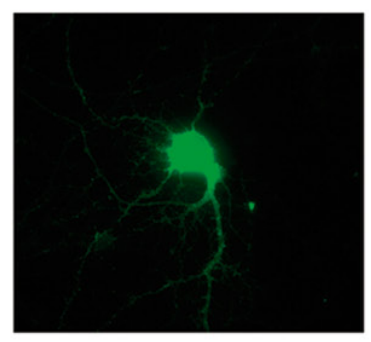

HA

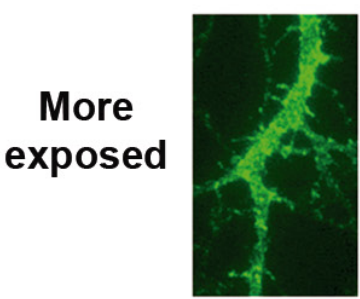

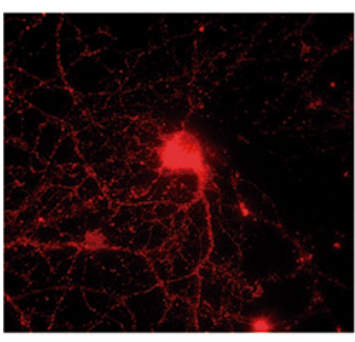

RTP801

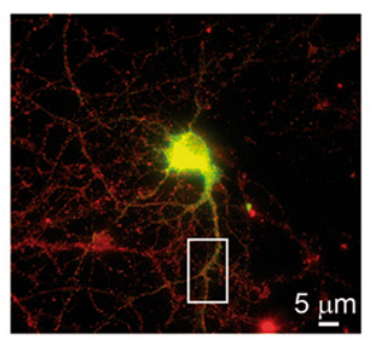

MERGE

Figure 2: NEDD4 and RTP801 interact physically. a. NEDD4 co-immunoprecipitates with RTP801 in cells exposed to DSP. HEK293 cells were co-transfected with empty vectors pCMS-eGFP and pCI-HA or with pCMS-eGFP-RTP801 and pCI-HANEDD4. Twenty-four hours post-transfection cells were exposed to cross-linker DSP for 2 hours at $4^{\circ} \mathrm{C}$ prior harvesting. RTP801 was immunoprecipitated and the samples were analyzed by Western Blotting. Membranes were probed with anti- NEDD4 and anti-RTP801 antibodies. Representative images are shown of at least three independent experiments. IP $=$ immunoprecipitation; IB $=$ immunoblot. $\mathbf{b}$. Endogenous RTP801 and NEDD4 interact in neuronal PC12 cells. NGF-differentiated PC12 cells were treated with $1 \mu \mathrm{M}$ epoxomicin for 2 hours. Then, cultures were exposed to DSP at $4{ }^{\circ} \mathrm{C}$ for 2 hours prior harvesting. RTP801 immunocomplexes were resolved in a Western Blotting. The membrane was incubated with anti-NEDD4 and anti-RTP801 antibodies. A representative image is shown of at least two independent assays. IP = immunoprecipitation; IB = immunoblot. c. NEDD4 and RTP801 co-localize in neurons. DIV 19 primary rat cortical neurons were transfected with pCI-HA-NEDD4. Forty-eight hours post-transfection, neurons were fixed and stained with antiRTP801 (in red) and anti-HA (in green). Scale bar, $5 \mu \mathrm{m}$. 
a

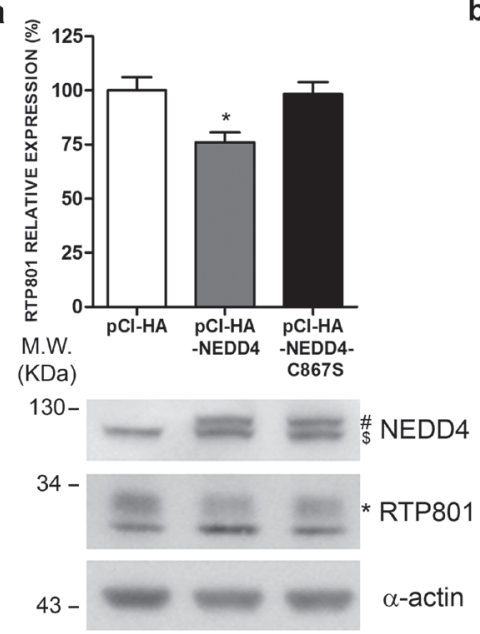

d

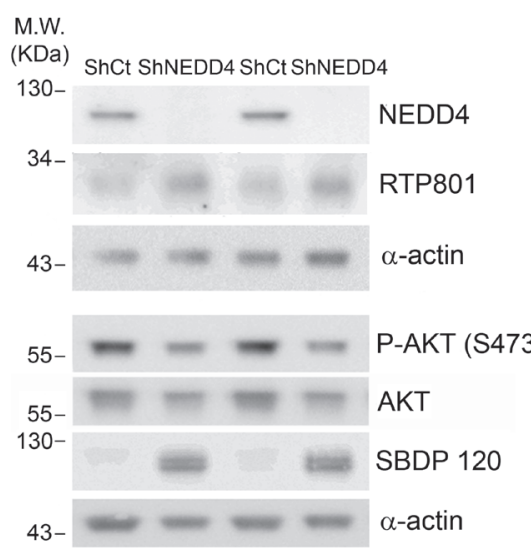

(a)
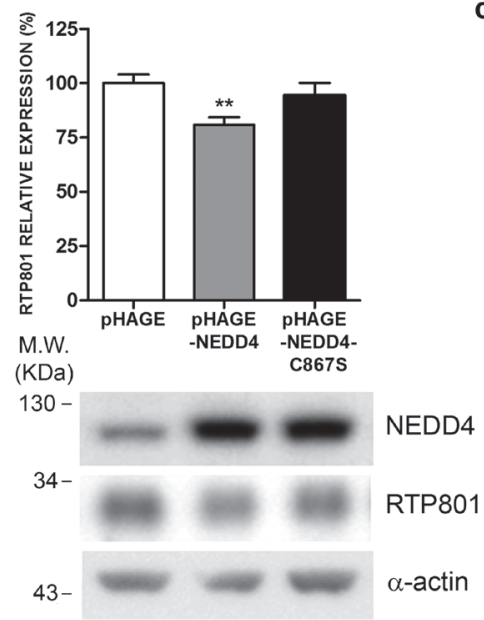

c

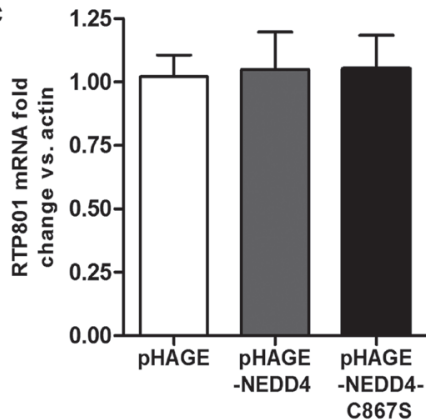

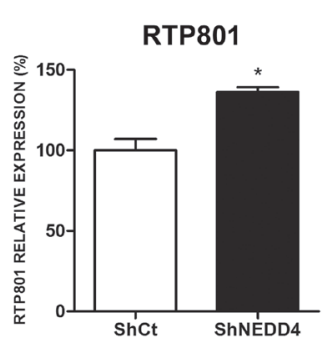

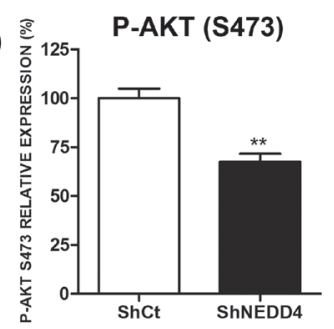

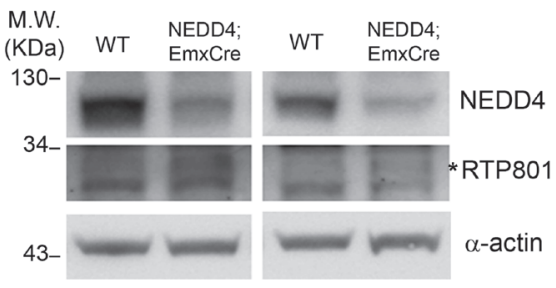

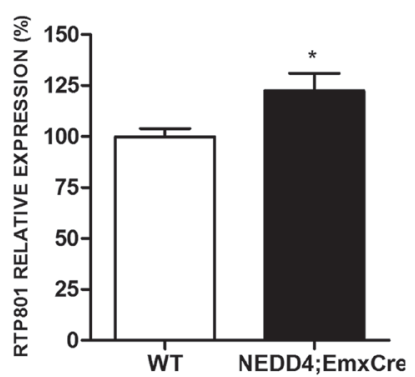

Figure 3: Ectopic NEDD4 regulates RTP801 in cultured cells. a. Ectopic NEDD4 decreases RTP801 protein levels in neuronal PC12 cells. NGF-differentiated PC12 cells were transfected with pCI-HA, pCI-HA-NEDD4 or pCI-HA-NEDD4-C867S constructs. Fortyeight hours post-transfection cultures were harvested and analyzed by Western Blot with anti-NEDD4, anti-RTP801 and anti- $\alpha$-actin antibodies. Representative immunoblots are shown along with densitometric quantification from at least three independent experiments. (\$ endogenous NEDD4, \# ectopic NEDD4, * specific band for RTP801). One-way ANOVA with Bonferroni multiple comparison test, ${ }^{*} P$ $<0.05$ versus pCI-HA. b. Ectopic NEDD4 decreases RTP801 protein levels in cortical neurons. DIV 8 rat primary cortical neurons were infected with lentiviruses containing the empty vector pHAGE, pHAGE-NEDD4 or pHAGE-NEDD4-C867S inactive mutant. Cell lysates were harvested 4 days later and analyzed by Western Blot with antibodies against RTP801, NEDD4 and $\alpha$-actin as loading control. The graph represents RTP801 densitometries of at least three independent experiments done in triplicate. One-way ANOVA with Bonferroni multiple comparison test, $* * P<0.01$ versus pHAGE. c. Ectopic NEDD4 does not affect RTP801 mRNA levels. DIV 8 cortical neurons were infected with lentiviruses containing the constructs pHAGE, pHAGE-NEDD4 or pHAGE-NEDD4-C867S. RNA was extracted 4 days later, and reverse transcription-qPCR was performed to quantify RTP801 transcripts. Results are displayed as RTP801 mRNA fold change respect to $\alpha$-actin mRNA levels. The graph shows values (mean \pm SEM) of three independent experiments. d. NEDD4 knockdown increases RTP801 protein levels and is detrimental for neurons. DIV 4 cortical neurons were infected with lentiviruses containing a scrambled shRNA $(\mathrm{ShCt})$ or a mix of three shRNA sequences against NEDD4 (ShNEDD4). Six days later, cells were harvested and analyzed by Western Blot. Membranes were incubated with NEDD4, RTP801, P-AKT (S473), AKT and $\alpha$-spectrin antibodies. The antibody against $\alpha$-actin was used as loading control. For Western blotting using the $\alpha$-spectrin antibody the caspase-cleaved fragment (spectrin breakdown product 120, SBDP120) is shown. Representative immunoblots are shown along with RTP801 and P-AKT (S473) densitometries (mean \pm SEM) of at least three independent experiments. Student's $t$-test, ${ }^{*} P<0.05$ and ${ }^{* *} P<0.01$ versus ShCt. e. NEDD4fff;Emx1Cre conditional knockout mice have elevated RTP801 protein levels in the cortex. Cortical lysates of 6-week old mice were analyzed by Western Blotting with antiNEDD4 and anti-RTP801 antibodies, and then reprobed with anti- $\alpha$-actin antibody as loading control. Representative immunoblots are shown along with RTP801 densitometries (mean \pm SEM) of at least three independent gels. All samples were immunoblotted in the same membrane, but some irrelevant lanes were deleted. (* Specific band for RTP801) Student's $t$-test, ${ }^{*} P<0.05$ versus WT. 

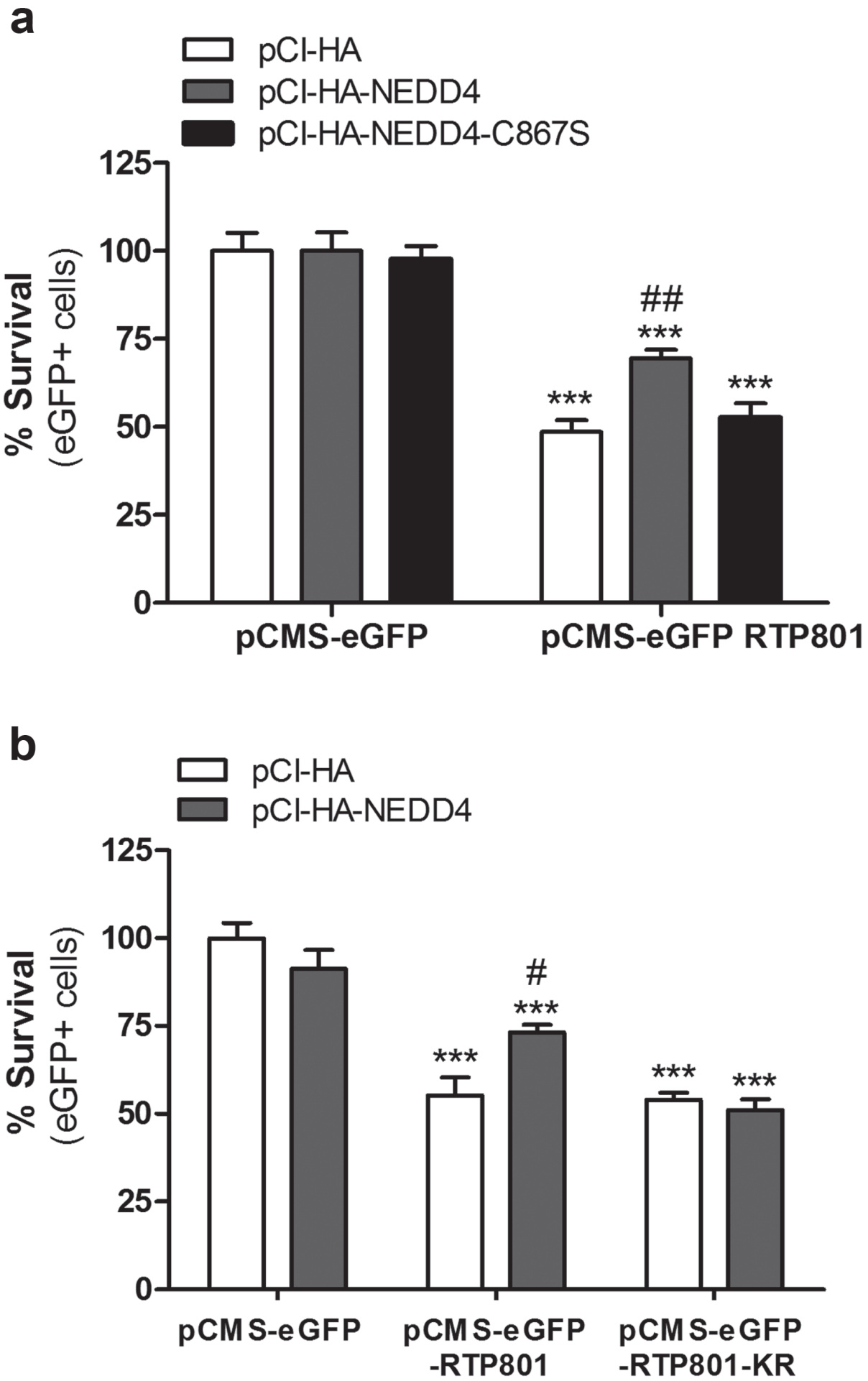

Figure 4: NEDD4 partially prevents from RTP801-induced cell death. a. Ectopic WT NEDD4 protects from RTP801-induced cell death. NGF-differentiated PC12 cells were co-transfected with pCI-HA, pCI-HA-NEDD4 or pCI-HA-NEDD4-C867S constructs, together with either pCMS-eGFP or pCMS-eGFP-RTP801. Cells were fixed 24 hours later and cell survival (eGFP+ cells) scored under fluorescence microscopy. The graph represents mean \pm SEM of at least three independent experiments in quadruplicates. One-way ANOVA with Bonferroni multiple comparison test, ${ }^{* * *} P<0.001$ versus $\mathrm{pCI}-\mathrm{HA} / \mathrm{pCMS}-\mathrm{eGFP},{ }^{\#} P<0.01$ versus $\mathrm{pCI}-\mathrm{HA} / \mathrm{pCMS}-\mathrm{eGFP}-\mathrm{RTP} 801$. b. NEDD4 does not protect from RTP801 KR mutant toxicity. NGF-differentiated PC12 cells were co-transfected with pCI-HA or pCIHA-NEDD4 together with pCMS-eGFP, pCMS-eGFP-RTP801 or pCMS-eGFP-RTP801-KR. Twenty-four hours later, cells were fixed and eGFP+ surviving cells scored using fluorescence microscopy. The graph represents mean \pm SEM of at least three independent experiments in quadruplicates. One-way ANOVA with Bonferroni multiple comparison test, ${ }^{* * *} P<0.001$ versus pCI-HA/pCMS-eGFP and ${ }^{\#} P<0.05$ versus $\mathrm{pCI}-\mathrm{HA} / \mathrm{pCMS}-\mathrm{eGFP}-\mathrm{RTP} 801$. 
whether 6-OHDA also influences the levels of NEDD4 in cellular cultures. In NGF-differentiated PC12 cells, 6-OHDA diminished NEDD4 protein levels but did not affect the levels of its mRNA. On the contrary, 6-OHDA elevated both mRNA and protein levels of RTP801 as reported previously (Figures 5a and 5b) $[6,8]$.

In order to investigate whether proteases such as caspases and calpains could be responsible for NEDD4 decrease, we pre-treated NGF-differentiated PC12 cells for one hour with $100 \mu \mathrm{M}$ of Z-VAD-FMK, a pan caspase inhibitor, or with $1 \mu \mathrm{M}$ of ALLN, a calpain inhibitor, and then, they were exposed to 6-OHDA. Both caspase and calpain independent inhibition partially prevented the loss of NEDD4 induced by 6-OHDA. The high levels of caspase-cleaved (SBDP120) and calpain cleaved-spectrin fragments (SBDP145) confirmed the toxicity of 6-OHDA (Figures 5c and 5d).

Taken together, these results indicate that caspase and calpain activation contribute to the loss of NEDD4 protein levels induced by 6-OHDA.

\section{Ectopic NEDD4 protects from 6-OHDA and prevents RTP801 elevation}

Since 6-OHDA decreased NEDD4 protein levels and this could be related with the elevation of RTP801, we first asked whether NEDD4 restitution could be protective against this neurotoxin. In fact, we chose to use the 6-OHDA model instead of over expressing alpha-synuclein because NEDD4 degrades alpha synuclein [15]. Thus, we overexpressed wild type or the inactive mutant of NEDD4 in NGF-differentiated PC12 cells and then exposed the cultures to 6-OHDA. By assessing cell survival, based on the number of eGFP+ cells with non-condensed or nonpyknotic nuclei (Hoechst staining), we concluded that ectopic WT NEDD4 partially prevented from 6-OHDAinduced cell death while the overexpression of the mutant had almost no effect (Figure 6a).

We next investigated whether the protection conferred by NEDD4 could be related with the modulation of RTP801 protein levels. WT NEDD4 or its inactive mutant NEDD4-C867S were overexpressed in NGFdifferentiated PC12 cells. After 32 hours, the cultures were exposed to 6-OHDA and cell lysates were analyzed by WB (Figure 6b). We observed a significant effect over RTP801 levels. Ectopic NEDD4 partially abrogated the 6-OHDA-induced levels of RTP801, in comparison to control or the NEDD4 inactive mutant.

Taken together, we conclude that NEDD4 protects from 6-OHDA toxicity and downregulates RTP801 induced by the toxin.

\section{NEDD4 loss is toxic due to RTP801 elevation in cortical neurons.}

We next investigated whether the toxicity of NEDD4 knockdown is mediated by RTP801 elevation in neurons. Rat primary cortical neurons were sequentially infected with lentiviral particles to knockdown RTP801 first and then NEDD4. WB analysis of the cell lysates showed that knocking down NEDD4 elevated RTP801 along with caspase-cleaved spectrin fragment SBDP120. Levels of phospho-S473-Akt and phospho-S6, as mTORC2 and mTORC1 activity readout respectively, were both reduced, although only P-S473-Akt was statistically significant. Indeed, knocking down RTP801 alone elevated the phosphorylation of Akt. Importantly, knocking down both RTP801 and NEDD4 abrogated RTP801 elevation and prevented the decrease of phospho-S473-Akt and phospho-S235/236-S6 (Figure 7).

These results conclude that NEDD4 has a robust functional relationship with RTP801 in the regulation of downstream targets mTOR and Akt.

\section{NEDD4 levels are diminished in nigral neurons in PD brains}

Previous studies showed that NEDD4 was upregulated in human nigral neurons containing Lewy Bodies (LBs) from patients diagnosed with Lewy bodies disease (LBD) [15]. However, only 15\% of nigral neurons contains alpha synuclein-reactive LBs [26]. For this reason, we extended the study to all nigral neurons in SNpc sections from sporadic PD patients. We immunostained 6 sporadic PD and 6 control cases with a specific antibody for NEDD4. We scored the number of nigral neurons, (visualized in brown due to neuromelanin pigment) that were positive for NEDD4 (in blue) versus total nigral neurons, in each case (Figure 8). We observed, independently of the presence of LBs, that the percentage of remaining nigral neurons stained for NEDD4 was lower in PD cases than in the age-matched controls (Figure $8)$. We did not observe staining in sections incubated only with the secondary antibody. This loss of NEDD4 expression in PD correlates well with the previously reported RTP801 elevation in nigral neurons $[6,8]$.

Taken together, these results show that NEDD4 is decreased in nigral neurons from PD patients that could well explain the previously reported RTP801 elevation in the same neuronal population $[6,8]$.

\section{DISCUSSION}

Here, we show that RTP801, a pro-apoptotic protein involved in neuron death in cellular and animal models of PD, is a novel substrate of NEDD4. NEDD4 polyubiquitinates RTP801 by conjugating K63 ubiquitin 
a

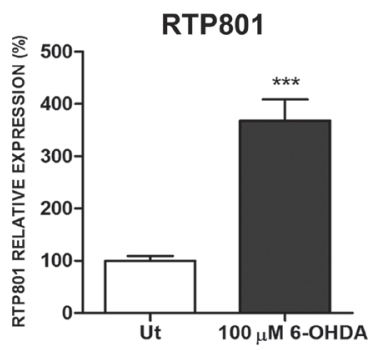

b

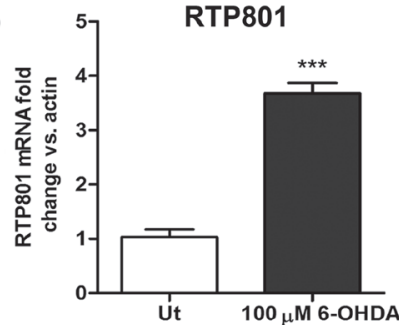

C

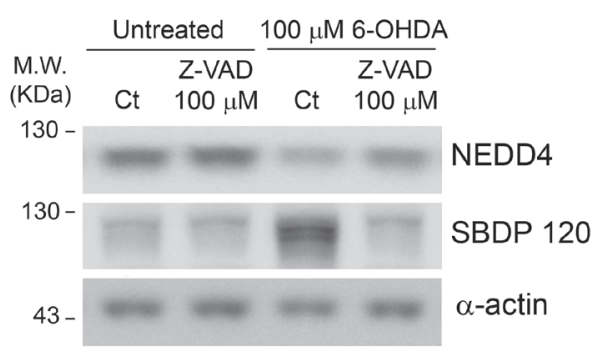

d

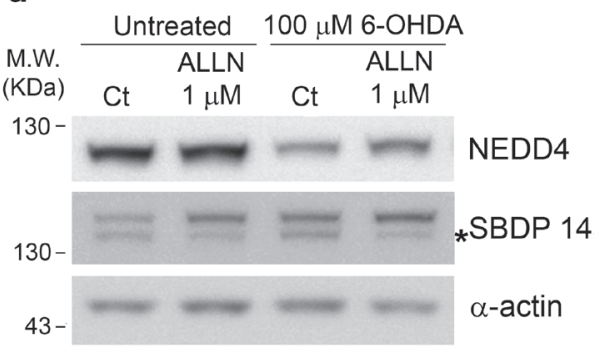

NEDD4
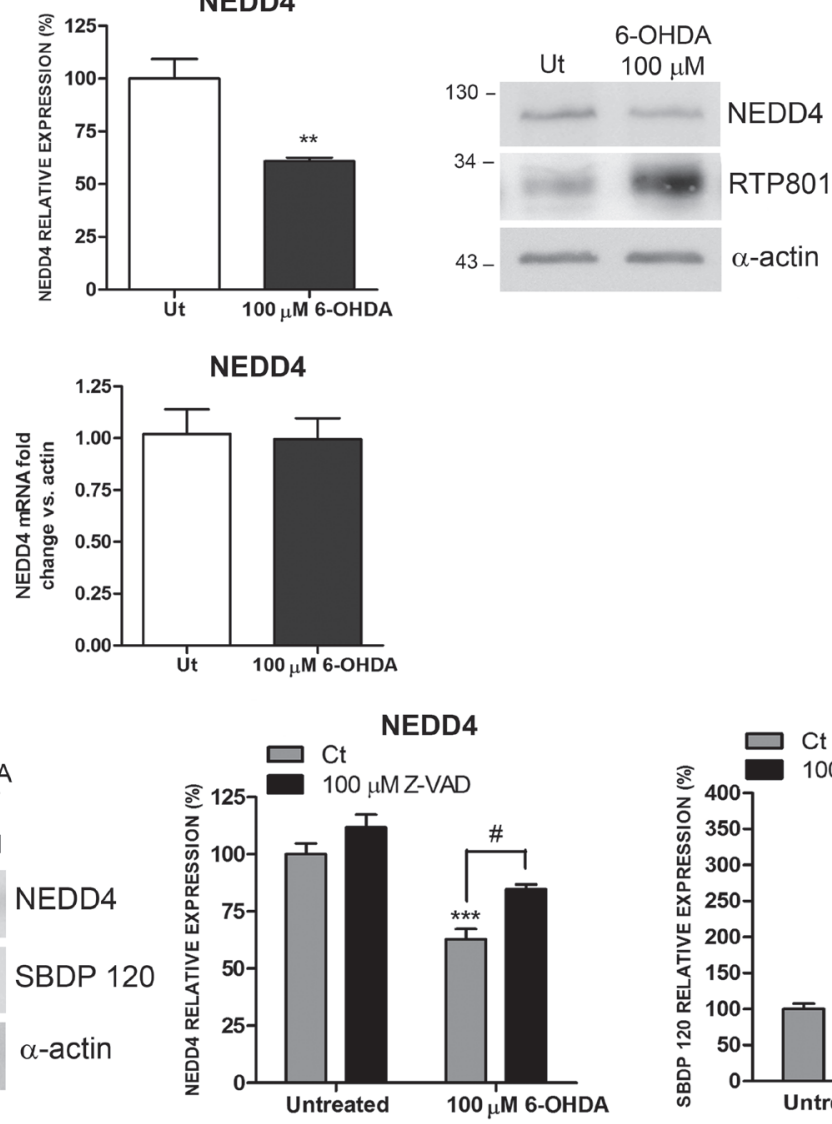

NEDD4

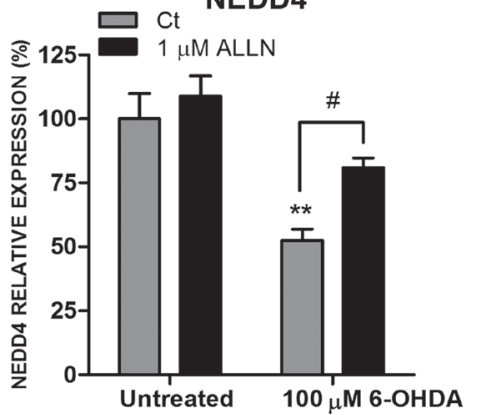

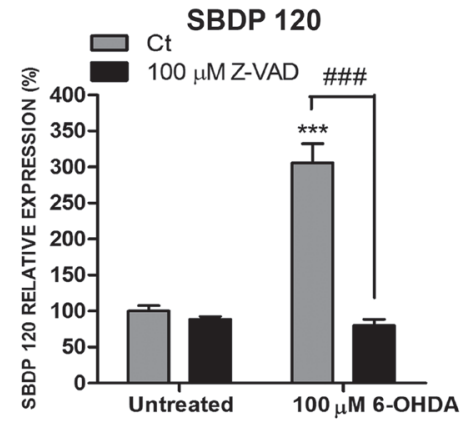

SBDP 145

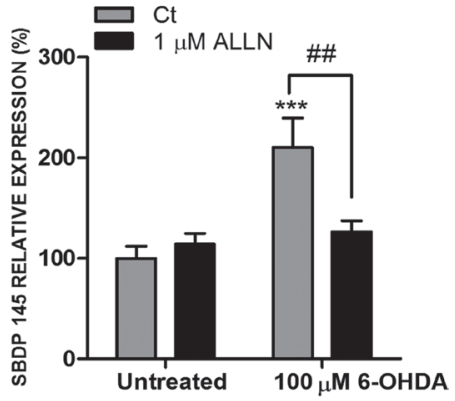

Figure 5: NEDD4 is decreased in a 6-OHDA PD cellular model. a. NEDD4 protein levels are diminished in 6-OHDAtreated neuronal PC12 cells. NGF-differentiated PC12 cells were exposed to $100 \mu \mathrm{M}$ 6-OHDA for 16 hours prior harvesting. Cell lysates were analyzed by Western Blot with antibodies against NEDD4, RTP801, as well as $\alpha$-actin antibody as loading control. Representative immunoblots are shown along with densitometries represented as mean \pm SEM of at least three independent experiments. Student's $t$-test, ${ }^{* *} P<0.01$ and $* * * P<0.001$ versus Ut (Untreated). b. NEDD4 mRNA levels are not modified in 6-OHDA-treated neuronal PC12 cells. NGF-differentiated PC12 cells were exposed to $100 \mu \mathrm{M}$ 6-OHDA for 8 hours. RNA was extracted and reverse transcription-qPCR was performed. The graphs show values (mean \pm SEM) of three independent experiments. Student's $t$-test, $* * * P<0.001$ versus ut (untreated). c. NEDD4 is cleaved by caspases after 6-OHDA exposure. Neuronal PC12 cells were treated with $100 \mu$ M Z-VAD-FMK pan-caspase inhibitor for 1 hour prior to 6-OHDA exposure. Sixteen hours later, cultures were harvested and analyzed by Western Blot. Membranes were incubated with antibodies against NEDD4 and $\alpha$-spectrin as well as an $\alpha$-actin antibody as loading control. Graphs represent mean \pm SEM of NEDD4 and caspase-cleaved $\alpha$-spectrin fragment (spectrin breakdown product $120 \mathrm{KDa}$, SBDP 120) densitometric quantification of at least three independent experiments. One-way ANOVA with Bonferroni multiple comparison test, $* * * P<0.001$ versus Untreated/Ct and ${ }^{\#} P<0.05,{ }^{\#} P<0.001$ versus $100 \mu \mathrm{M}$ 6-OHDA/Ct. d. NEDD4 is cleaved by calpains after 6-OHDA exposure. Neuronal PC12 cells were treated with $1 \mu \mathrm{M}$ ALLN calpain inhibitor for 1 hour prior to 6-OHDA exposure. Sixteen hours later, cultures were harvested and subjected to Western Blot. Membranes were incubated with anti-NEDD4 and anti- $\alpha$-spectrin antibodies and with anti- $\alpha$-actin as loading control. Graphs represent mean \pm SEM of NEDD4 and calpain-cleaved $\alpha$-spectrin fragment (spectrin breakdown product $145 \mathrm{KDa}$, SBDP 145) densitometric quantification of at least three independent experiments. One-way ANOVA with Newman-Keuls multiple comparison test, ${ }^{* *} P<0.01,{ }^{* * *} P<0.001$ versus Untreated/Ct and ${ }^{\#} P<0.05,{ }^{\# \#} P<0.001$ versus $100 \mu \mathrm{M} 6-\mathrm{OHDA} / \mathrm{Ct}$. 


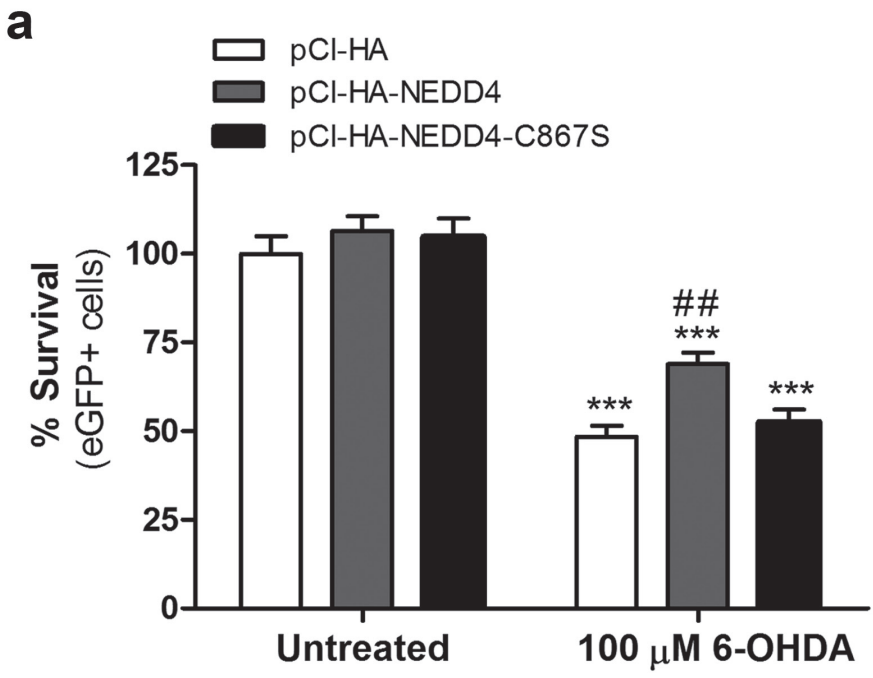

b

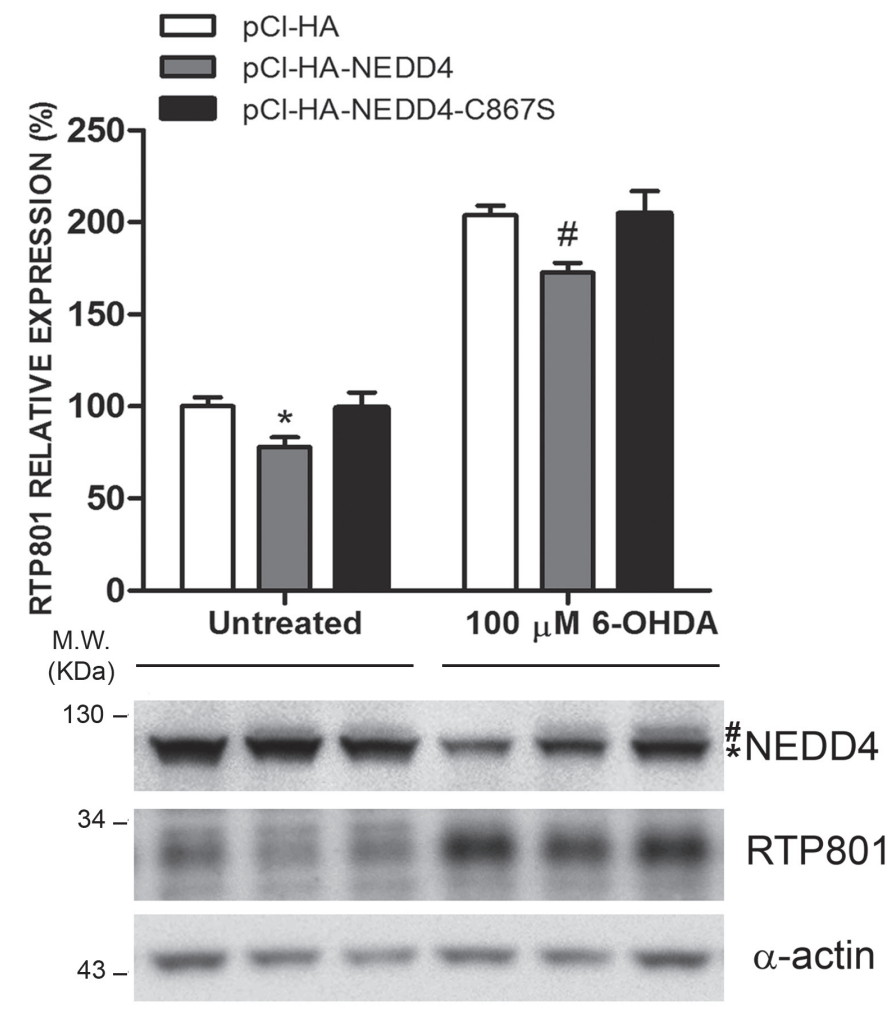

Figure 6: NEDD4 protects from 6-OHDA-induced cell death and decreases RTP801 elevation. a. Ectopic NEDD4 WT partially prevents from 6-OHDA-induced cell death. NGF-differentiated PC12 cells were co-transfected with pCI-HA/pCMS-eGFP, pCIHA-NEDD4/pCMS-eGFP or pCI-HA-NEDD4-C867S/pCMS-eGFP vectors with a 4:1 ratio. Thirty-two hours later, cell cultures were exposed to $100 \mu \mathrm{M}$ 6-OHDA for 16 hours. Then, eGFP+ surviving cells were scored under fluorescence microscopy. The graph shows mean \pm SEM of at least three independent experiments done in quadruplicate. One-way ANOVA with Bonferroni multiple comparison test, $* * * P$ $<0.001$ versus Untreated/pCI-HA and ${ }^{\# \prime} P<0.01$ versus $100 \mu \mathrm{M}$ 6-OHDA/pCI-HA. b. Ectopic NEDD4 reduces RTP801 elevation after 6-OHDA exposure. NGF-differentiated PC12 cells were transfected with pCI-HA, pCI-HA-NEDD4 or pCI-HA-NEDD4-C867S. Thritytwo hours later, cell cultures were exposed to $100 \mu \mathrm{M}$ 6-OHDA for 16 hours and cell lysates were analyzed by Western Blot. Membranes were incubated with antibodies against NEDD4, RTP801 and $\alpha$-actin as loading control (* endogenous NEDD4, \# ectopic NEDD4). Low signal of ectopic NEDD4 maybe due low sensitivity of NEDD4 antibody towards the human NEDD4 protein, as compared to endogenous rat NEDD4. Representative immunoblots are shown along with RTP801 densitometric normalized quantification (mean \pm SEM) from at least three independent experiments. One-way ANOVA with Bonferroni multiple comparison test, ${ }^{*} P<0.05$ versus Untreated/pCI-HA, ${ }^{*} P$ $<0.05$ versus $100 \mu \mathrm{M} 6-\mathrm{OHDA} / \mathrm{pCI}-\mathrm{HA}$. 
chains. This type of posttranslational modification is associated with the lysosomal pathway. NEDD4 and RTP801 interact physically in HEK293 and in NGFdifferentiated PC12 cells. NEDD4 also regulates RTP801 protein levels in both cultured cells and in a conditional NEDD4 knockout mouse model. Moreover, NEDD4 protein levels are diminished in remaining nigral neurons in human sporadic PD brains. Furthermore, we provide evidence that NEDD4 is protective against ectopic RTP801 toxicity by ubiquitinating it. We found that 6-OHDA neurotoxin exposure decreased dramatically NEDD4 protein levels and its restoration abrogated 6-OHDA-induced cell death. Ectopic NEDD4 also prevented RTP801 protein elevation induced by 6-OHDA. Finally, NEDD4 loss of function toxicity was mediated by RTP801 by inactivating mTOR/Akt kinases.

The finding that RTP801 is also degraded by the lysosomal system in neurons was yet not known. In lymphocytes treated with dexamethasone, RTP801/ REDD1 mediated the fusion of lysosomes with autophagosomes, and therefore it mediated autophagy [27]. Interestingly, mTORC1 translocates to the lysosomes surface via the Rag-ragulator complex to be activated [28]. One can speculate that NEDD4 targeting RTP801 to the lysosomal degradation pathway is necessary for lysosomal mTOR translocation/activation; therefore an elevation of RTP801 could be deleterious for this process.

Our previous studies confirmed that RTP801 was mostly degraded by the proteasome. This is in line with the evidence relating proteins with short half-lives more prone to be degraded by the proteasome. However, here we found a pool of RTP801 that is degraded by the lysosomal pathway. Both proteasome and lysosome systems seem to be impaired in PD [29, 30]. Thus, based on our previous and current results, the deregulation of both processes could be contributing importantly to RTP801 elevation in the pathogenesis of PD.

We show that NEDD4 ubiquitinates RTP801, preferentially with K63-ubiquitin chains. K63-ubiquitin chains have a low affinity for the $26 \mathrm{~S}$ proteasome subunit and they rather bind other soluble proteins, such as ESCRT-0 (Endosomal Sorting Complex Required for Transport) and its components, STAM and Hrs that block the interaction with S26 proteasomal subunit [31]. Therefore they are targeted to the lysosomal pathway. On the contrary, K48-ubiquitin chains have more affinity for $\operatorname{Rad} 23$ proteins, especially hHR23B, that promote proteasome binding [31].

NEDD4 binds proteins with proline rich motifs [32] or phospho-serines or phospho-threonines [33] via its WW binding domains. Indeed, RTP801 protein sequence has a proline rich region in the fragment close to the $\mathrm{N}$-terminus that contains several serines and threonines. Interestingly, threonine residues 23 and 25 fit the consensus sequence for GSK3b kinase and could be therefore, phosphorylated [34].
To date only three E3 ligases have been described to regulate RTP801: CUL4A- DDB1-ROC1-b-TRCP E3 ligase complex, HUWE-1 and parkin. CUL4A-DDB polyubiquitinates RTP801 and mediates its proteasomal degradation in a GSK3 phosphorylation dependent manner [34]. However, other authors could not confirm these results by using a chemical inhibitor of all cullin E3 ligases [35].

We recently found that parkin polyubiquitinates RTP801 and mediates its proteasomal degradation [8]. Parkin dysfunction, due to mutations or oxidative/ nitrosative stress has been consistently linked to PD [3640]. Regarding NEDD4 involvement in PD, Tofaris and colleagues (2011) [15] first described the possible role of NEDD4 in the endosomal-lysosomal degradation of alphasynuclein in cellular models and in yeast. However in their work, whether NEDD4 activity was altered in PD, as it is the case with parkin, was not clear.

NEDD4 knockdown elevated RTP801 and caspasecleaved spectrin fragment SBDP120. Interestingly it also inactivated Akt by decreasing the levels of phosphorylation at the Serine-473 residue. All these events suggest that NEDD4 loss of function was toxic for neurons.

These signaling events were clearly observed in mature rat cortical neurons although they were not significant in the 6-week-old mice cortical lysates from the NEDD4f/f;Emx1Cre conditional knock out mice. These results suggest that in these mice, neuronal death could be masked due to compensatory mechanisms.

NEDD4 loss is toxic in cortical neurons and at least, it involves RTP801 elevation and inactivation of the mTOR/Akt kinases. In fact, knocking down RTP801 at the same time as NEDD4 prevented the loss of phospho-Akt and phospho-S6 and the appearance of caspase-cleaved spectrin fragments. NEDD4 has been related with Akt survival signaling. NEDD4 polyubiquitinates phosphoAkt at the plasma membrane to modulate Akt subcellular localization [41]; it binds to GRB10 to regulate IGF-1 and insulin signaling, including IGF1 receptor ubiquitination [42]. Interestingly, Grb10 is an mTORC1 substrate [43].

Hence, NEDD4 activity over RTP801, which in turn is a negative regulator of Akt and mTOR, would be crucial to prevent neurodegeneration.

Consistent with the toxicity induced by the loss of NEDD4 in cultured cells, we found in human PD brains that the percentage of NEDD4-stained nigral neurons were lower in PD cases than in age-matched controls. This result correlates with the previously reported elevation of RTP801 in nigral neurons in sporadic PD cases [6, 8]. However, Tofaris et al. (2011) [15] described that NEDD4 was strongly expressed in pigmented neurons from both LC and SN containing LBs, as a neuroprotectective mechanism against neurodegeneration [15]. According to literature, only $15 \%$ of the remaining nigral neurons contains LB [26]. The fact that we considered all the remaining nigral neurons could explain the differences 


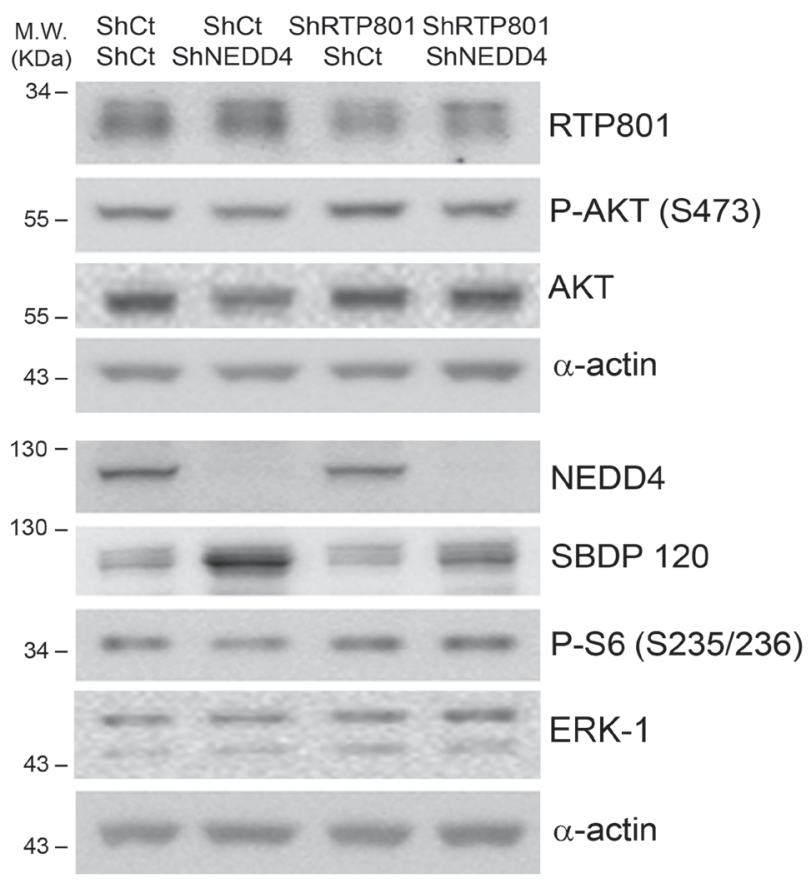

RTP801

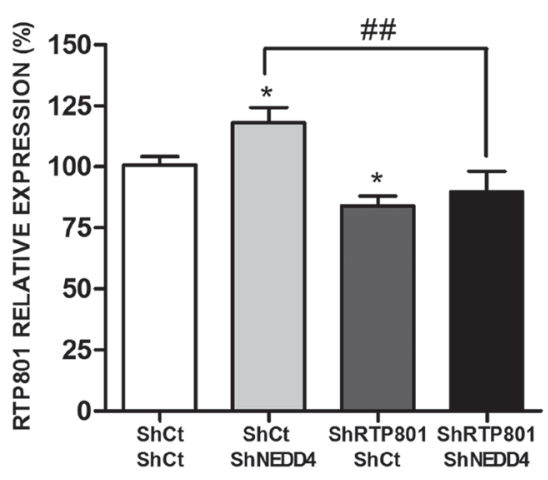

P-AKT (S473)

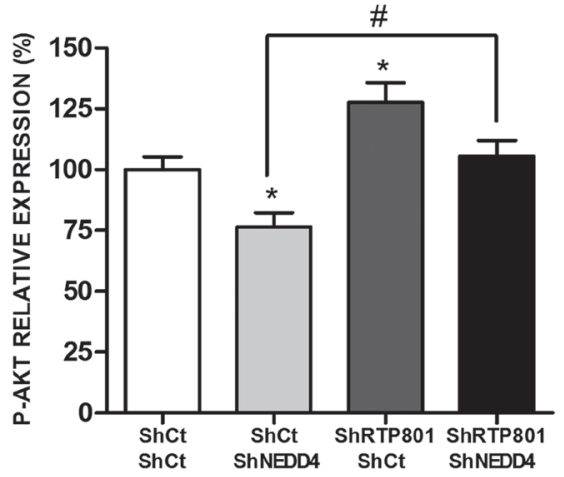

SBDP 120

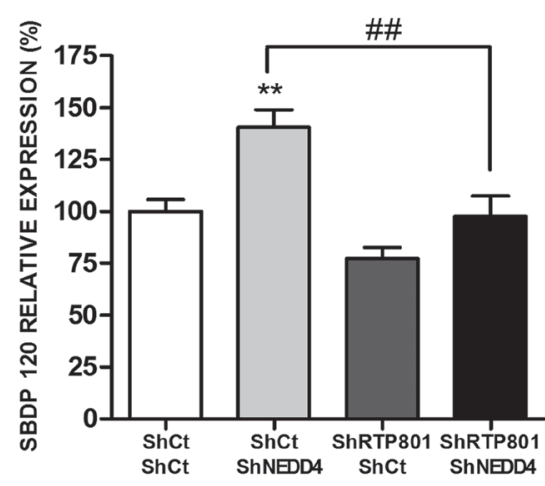

P-S6 (S235/236)

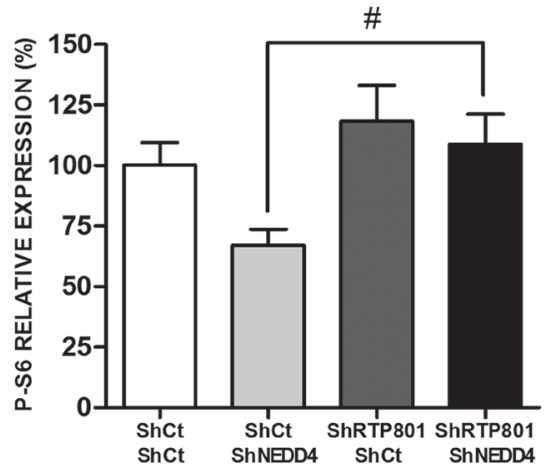

Figure 7: NEDD4 knockdown toxicity is dependent on RTP801 protein. DIV 5 primary rat cortical neurons were infected with lentiviruses containing a scrambled shRNA (ShCt) or a shRNA against RTP801 (ShRTP801). Two days later, neurons were transduced with a mix of three shRNA sequences against NEDD4 (ShNEDD4) or the corresponding control shRNA (ShCt). Cell lysates were analyzed 4 days later by Western Blot. Membranes were incubated with RTP801, NEDD4, P-AKT (S473), AKT, P-S6 (S235/236), ERK1/2 and $\alpha$-spectrin antibodies, and with $\alpha$-actin antibody as loading control. For $\alpha$-spectrin the caspase-cleaved fragment (spectrin breakdown product 120, SBDP120) is shown. Representative immunoblots are shown along with densitometries (mean \pm SEM) of at least two independent experiments done in triplicate. One-way ANOVA with Newman-Keuls multiple comparison test, $* P<0.05$, $* * P<0.01$ versus ShCt/ShCt and ${ }^{\#} P<0.05,{ }^{\#} P<0.01$ versus ShCt/ShNEDD4. 

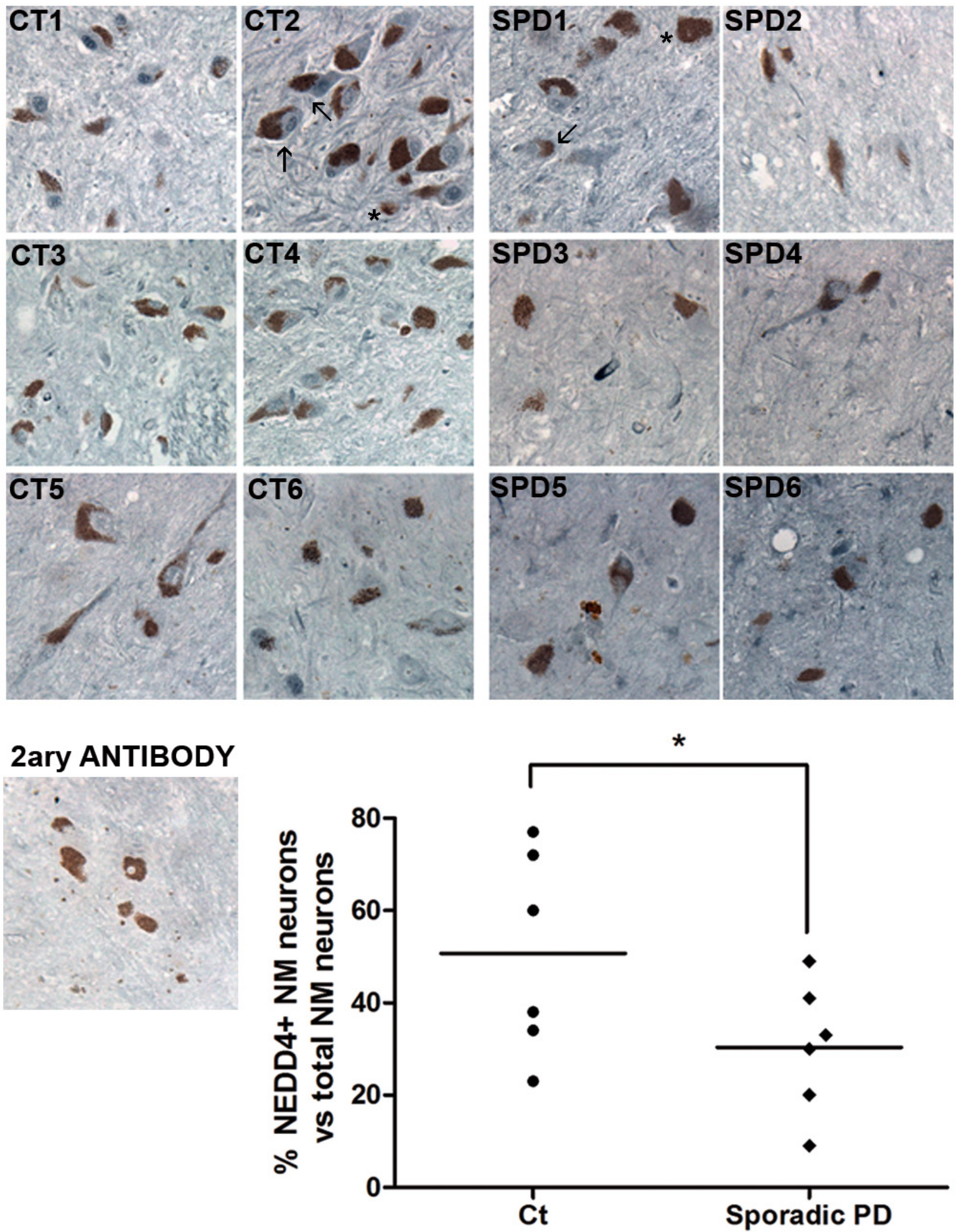

Figure 8: NEDD4 is decreased in pigmented nigral neurons from sporadic PD patients. SNpc human postmortem sections from control individuals (CT1-CT6) and sporadic PD patients (SPD1-SPD6) were immunostained for NEDD4 (grey-blue). Note the presence of neuromelanin (NM) granules (brown) within the somas of dopaminergic neurons. Neuromelanin-positive (NM+) neurons were scored positive for NEDD4 when detectable staining was observed. For each case, the percentage of NM+ and NEDD4+ neurons versus total $\mathrm{NM}+$ neurons was represented in the graph. A control section was immunostained with no primary antibody as negative control. Arrows indicate some of the positive cells stained for NEDD4 and asterisks show some of the negative cells for NEDD4. Counting was performed in blind conditions. Student's $t$-test, $* P<0.05$. 
between these two works. Whether RTP801 is elevated in LC has not been yet explored.

In conclusion, here we show that the HECT E3 ligase NEDD4 mediates the ubiquitination of proapoptotic protein RTP801 and its lysosomal degradation. We observed that NEDD4 knockdown is toxic for neurons and this toxicity is counteracted when RTP801 elevation is abrogated. Moreover, the levels of NEDD4 in the remaining nigral neurons in human PD brains are diminished. These nigral neurons also present elevated levels of RTP801. Thus, the loss of NEDD4 in PD could be leading to neurodegeneration at least in part, by contributing to RTP801 toxic accumulation.

\section{MATERIALS AND METHODS}

\section{Antibodies, plasmids and materials}

Rabbit polyclonal antibody against RTP801 was purchased from Proteintech Group Inc (Chicago, IL, USA). Rabbit polyclonal anti-NEDD4 (used for WB), rabbit polyclonal anti-ERK1/2 and mouse monoclonal anti-GFP antibodies were obtained from Santa Cruz Biotechnology (Dallas, TX, USA). Anti-HA antibody (used for WB), anti-P-Akt (S473), anti-P-S6 (S235/236) and anti-Akt rabbit polyclonal antibody were purchased from Cell Signaling Technology (Danvers, MA, USA). Mouse monoclonal antibody against HA-tag (used for immunofluorescence) was obtained from Sigma-Aldrich (Sant Louis, MO, USA). Ubiquitin (FK2) antibody was purchased from Enzo Life Sciences (Farmingdale, NY, USA). Rabbit polyclonal antibody against NEDD4 and GAPDH (used for immunohistochemistry) were from Abcam (Cambridge, UK). The antibody against $\alpha$ IIspectrin was purchased from Merck Millipore (Billerica, MA, USA). Anti- $\alpha$-actin antibody was obtained from MP Biomedicals (Santa Ana, CA, USA). Horseradish peroxidase-conjugated goat anti-mouse and anti-rabbit secondary antibodies were obtained from Pierce Thermo Fisher Scientific (Rockford, IL, USA). Goat anti-mouse and anti-rabbit secondary antibodies conjugated to Alexa 488 or Alexa 568 were purchased from Thermo Fisher Scientific (Waltham, MA, USA).

pCMS-eGFP-RTP801 and pCMS-eGFP-RTP801

$\mathrm{KR}$ constructs were generated as previously described $[6,8]$. pCI-HA-NEDD4 and pcDNA3 HA-ubiquitin were purchased from Addgene (Cambridge, MA, USA). The construct pCI-HA was a kind gift from Dr. Joan Massagué (Memorial Sloan Kettering Cancer Center, New York, NY, USA). pHAGE and pHAGE-NEDD4 constructs were kindly provided by Dr.Heng-Ye Man (Boston University, MA, USA). pRK5-HA-ubiquitin-K48 and pRK5-HAubiquitin-K63 were a kind gift from Dr. Bernat Crosas (Molecular Biology Institute of Barcelona, CSIC, Spain).
The lentivirus packaging plasmids pMDLg/pRRE, pRSV-Rev and pMD2.G used to knockdown RTP801 were obtained from Addgene. The lentivirus packaging plasmids pHDM-Tat1b, pRC/CMV-Rev1b, pHDMHgpm 2 and pHDM-G used to overexpress NEDD4 were kindly provided by Dr. Heng-Ye Man. All vectors were validated by DNA sequencing.

Epoxomicin, MG132, cycloheximide, Z-VADFMK, and ALLN were purchased from Calbiochem Merck Millipore (Billerica, MA, USA), chloroquine and CHAPS were obtained from Sigma-Aldrich, and 6-OHDA was purchased from Tocris Bioscience (Bristol, UK).

\section{Directed mutagenesis}

pCI-HA-NEDD4-C867S and pHAGE-NEDD4C867S constructs were obtained by mutating the catalytic cysteine (C) 867 to serine (S) of pCIHA-NEDD4 and pHAGE-NEDD4 vectors. The QuickChange Lightning II multi site-directed mutagenesis kit (Agilent Technologies, Santa Clara, CA, USA) was used with the following primer: 5'-GTC CAGGCGATTAAAACTGGTATGAGCTCTTGGCA-3'. All new constructs were validated by DNA sequencing.

\section{ShRNA production}

Scrambled control ShRNA (ShCt) and ShRTP801 were generated as previously described [6], based on the following sequences: ShCt, 5'GTGCGTTGCTAGTACCAAC-3', ShRTP801, 5'-AAGACTCCTCATACCTGGATG-3' (specific for human, rat and mouse RTP801). The two ShRNA were inserted into the lentiviral vector pLL3.7 (Addgene).

\section{NEDD4f/f;Emx1Cre mice}

In order to generate the forebrain specific NEDD4 conditional knockout mouse, Nedd4 ${ }^{\mathrm{f} / \mathrm{f}}$ mice [13] were crossed with EmxlCre mouse [24]. EmxlCre mice express Cre recombinase in the radial glia cells, resulting in floxrecombination of floxed target genes in glutamatergic neurons and glia cells in the cerebral cortex.

\section{Cell culture}

PC12 cells were maintained and differentiated with NGF as previously described [18]. For NGF treatment, cells were grown in RPMI 1640 medium (Thermo Fisher Scientific) supplemented with $1 \%$ heat-inactivated horse serum (Sigma-Aldrich), penicillin/streptomycin (Thermo Fisher Scientific) and $50 \mathrm{ng} / \mathrm{ml}$ recombinant human $\beta$-NGF (Alomone Labs, Jerusalem, Israel) for 7-8 days in a $7,5 \% \mathrm{CO}_{2}$ atmosphere at $37^{\circ} \mathrm{C}$. Medium was changed 
every other day and before transfection.

HEK293 cells were cultured in DMEM medium supplemented with $10 \%$ fetal bovine serum and penicillin/ streptomycin (all from Thermo Fisher Scientific) in a 5\% $\mathrm{CO}_{2}$ atmosphere at $37^{\circ} \mathrm{C}$.

Rat primary cortical cultures were prepared as previously reported [19]. Briefly, cortex from embryonic (E18) Sprague-Dawley rats were dissected out, dissociated and plated at a density of 250 cells $/ \mathrm{mm}^{2}$ on poly-L-lysinecoated (Sigma-Aldrich) coverslips or at a density of 700 cells $/ \mathrm{mm}^{2}$ on poly-L-lysine-coated plates. Neurons were maintained in neurobasal medium with B27 and $2 \mathrm{mM}$ GlutaMAX (all from Gibco). However, neurons plated on coverslips were initially seeded with MEM medium supplemented with $100 \mathrm{mM}$ pyruvic acid (Gibco), 20\% glucose (Sigma-Aldrich) and 10\% heat-inactivated horse serum.

\section{Lentiviral preparation}

Lentiviral particles were produced by transient transfection with Lipofectamine 2000 (Thermo Fisher Scientific) in HEK293 cells. 72 hours posttransfection, cell medium was collected, centrifuged to remove debris and filtered through $0.45 \mu \mathrm{m}$-pore filters (Thermo Scientific). Lentiviruses were concentrated by ultracentrifugation and resuspended in sterile PBS Ca2+/ $\mathrm{Mg} 2+$ (Fisher Scientific). Viral titer was assessed by transduction of several viral dilutions.

\section{Transfection, treatments and viral infection}

Primary rat cortical neurons, neuronal PC12 cells or HEK293 cells were transfected with Lipofectamine 2000 (Thermo Fisher Scientific) in accordance with the manufacturer's instructions. Neuronal PC12 cells transfection rate is around $15-20 \%$ and $\%$ of neuronal viral transduction is at least a $60 \%$.

6-OHDA treatments were performed after 5-6 days of differentiation in NGF-treated PC12 cells. Medium was replaced right before exposure to the toxin.

Rat cortical neurons were infected at DIV 4 or at DIV 7 with lentiviral particles containing an ShRNA construct against rat NEDD4 (ShNEDD4) or a scrambled control sequence $(\mathrm{ShCt})$, both purchased from Santa Cruz Biotechnologies. In the case of lentiviral particles containing ShCt and ShRTP801, cortical neurons were transduced at DIV 5. Lentiviral particles containing pHAGE, pHAGE-NEDD4 or pHAGE-NEDD4-C867S were infected at DIV 7. In all cases, neurons were infected at a multiplicity of infection (MOI) of 1 .

\section{In vitro ubiquitination assay}

The Ubiquitinylation Kit (Enzo Life Sciences) was used to perform the assay. Manufacturer's instructions were followed with minor modifications. Shortly, 1 $\mathrm{mM}$ dithiothreitol in $20 \mathrm{mM}$ Tris- $\mathrm{HCl} \mathrm{pH} \mathrm{7.5,} 20 \mathrm{U} /$

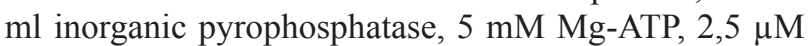
biotinylated ubiquitin, 0,1 $\mu \mathrm{M}$ His6-tagged recombinant human ubiquitin-activating enzyme E1, His6-tagged recombinant human ubiquitin-conjugating enzyme UbcH5b E2 (Enzo Life Sciences), recombinant human NEDD4 protein (Sigma Aldrich) and N-terminal GSTtagged recombinant human RTP801 full-length protein (Novus Biologicals, Littleton, CO, USA) were added to the reaction buffer, as specified in each condition, and then incubated at $37^{\circ} \mathrm{C}$ for $90 \mathrm{~min}$. Next, RTP801 was immunoprecipitated using an anti-RTP801 antibody and equal volumes of each sample were analyzed by WB. Membranes were probed for biotinylated ubiquitin using Avidin/Biotin-Horseradish Peroxidase (Ultra-sensitive ABC staining kit; Thermo Fisher Scientific) or for RTP801.

\section{Western blot}

Whole cell extracts and NEDD4f/f;Emx 1 Cre cortical lysates were collected and processed as previously described $[6,44]$. Chemiluminiscent images were acquired using a LAS-3000 Imager (Fujifilm, Valhalla, NY, USA) and quantified by computer-assisted densitometric analysis (ImageJ).

\section{Immunoprecipitation and ubiquitination assay}

Cell extracts were collected with RIPA buffer (50 mM Tris- $\mathrm{HCl}$ pH 7.4, 150 mM NaCl, 1\% NP-40 (SigmaAldrich), 1\% sodium deoxycholate (Sigma-Aldrich) and $0.1 \%$ sodium dodecyl sulfate (Sigma-Aldrich), containing mini cOmplete protease inhibitor (Roche Diagnostics Corporation, Indianapolis, IN, USA). Lysates were further solubilized by sonication and centrifuged for $10 \mathrm{~min}$ at $13000 \mathrm{~g}$ to remove insolubilities. Equal amounts of total protein were incubated overnight $(\mathrm{O} / \mathrm{N})$ on rotation at $4^{\circ} \mathrm{C}$ with protein A-agarose beads (Santa Cruz Biotechnology) and with the corresponding antibody or a normal immunoglobulin (Santa Cruz Biotechnology) as a negative control. Then the beads were centrifuged and washed four times with RIPA buffer and the immunocomplexes were collected and analyzed by WB.

\section{Co-immunoprecipitation}

The cross-linking agent DSP (Thermo Fisher Scientific) was applied to cell cultures according 
Table 1: Human brain samples information.

\begin{tabular}{|l|l|l|l|l|l|}
\hline $\begin{array}{l}\text { PATIENT } \\
\text { ID }\end{array}$ & $\begin{array}{l}\text { CLINICAL } \\
\text { DIAGNOSIS }\end{array}$ & $\begin{array}{l}\text { ANATOMO-PATHOLOGICAL } \\
\text { DIAGNOSIS }\end{array}$ & GENDER & AGE & $\begin{array}{l}\text { TIME } \\
\text { POSTMORTEM } \\
\text { (hours) }\end{array}$ \\
\hline CT1 & Control & NFT II + alpha synuclein in olfactory bulb & Male & 64 & 10 \\
\hline CT2 & Control & AgD I & Male & 83 & 13 \\
\hline CT3 & Control & $\begin{array}{l}\text { Cerebral metastasis from lung cancer + } \\
\text { NFTI-II }\end{array}$ & Female & 56 & 14 \\
\hline CT4 & Control & $\begin{array}{l}\text { Spine thrombosis D + cerebellar ictus +bulb, } \\
\text { NFT III }\end{array}$ & Female & 86 & 4 \\
\hline CT5 & Control & iLBD Braak 1, NFT I-II, SVD & Male & 78 & 6 \\
\hline CT6 & Control & Minimum AgD I, with patched gliosis & Male & 76 & 11,5 \\
\hline SPD1 & PD & LBD Braak 4, ARP I B & Male & 71 & 5 \\
\hline SPD2 & PD & LBD Braak 4 + NFT Braak II & Male & 77 & 12 \\
\hline SPD3 & PD & $\begin{array}{l}\text { LBD Braak 4, ARP II/A, discreet CAA, } \\
\text { SVD }\end{array}$ & Male & 88 & 15 \\
\hline SPD4 & PD & LBD 4-5 + ARP IIA & Female & 83 & 4 \\
\hline SPD5 & PD & LBD Braak 5 + ARP III B + CAA mod & Male & 74 & 8 \\
\hline SPD6 & PD & LBD Braak 5 + AgD & Male & 81 & 5 \\
\hline
\end{tabular}

$\mathrm{CT}=$ control individuals, $\mathrm{SPD}=$ sporadic PD patients LBD: Lewy body disease, stages based on Braak et al. (2003): 1-6. ARP: Alzheimer-type pathology: classification of the neurofibrillary pathology NFT based on Braak et al., (2006) (I-VI) and the neuritic plaques based on CERAD criteria (A-C), CAA amyloid angiopathy. PMD: postmortem delay (in hours). AgD: Argyrophilic grain Disease, SVD Small Vessel Disease.

to manufacturer's instructions. Then, cell extracts were collected with cell lysis buffer (Cell Signaling Technology). Equal amounts of total protein were incubated overnight $(\mathrm{O} / \mathrm{N})$ at $4^{\circ} \mathrm{C}$ on rotation with protein A-agarose beads (Santa Cruz Biotechnology) and with RTP801 antibody or a normal immunoglobulin (Santa Cruz Biotechnology). Beads were centrifuged, washed

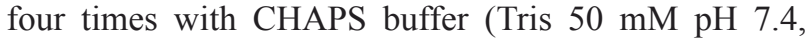
$\mathrm{NaCl} 150 \mathrm{mM}, \mathrm{MgCl}_{2} 10 \mathrm{mM}$, CHAPS 0,4\%), and the immunocomplexes were resolved in a WB.

\section{Quantitative reverse transcription-PCR}

Total RNA was isolated from NGF-differentiated PC12 cells or rat primary cortical neurons with the High Pure RNA Isolation Kit (Roche Diagnostics Corporation, Indianapolis, IN, USA). cDNA reverse transcription from total RNA was performed by using the Transcriptor First Strand cDNA synthesis Kit (Roche Diagnostics Corporation). The following primers were used for quantitative PCR amplification: RTP801 forward primer, 5'-GCTCTGGACCCCAGTCTAGT-3'; RTP801 reverse primer, 5'-GGGACAGTCCTTCAGTCCTT-3'; NEDD4 forward primer, 5' GGACGAGGTATGGGAGTTCT-3'; NEDD4 reverse primer, 5'- CTCCACTCATCGGGTCATAC-3'; $\alpha$-actin forward primer, 5'-GGGTATGGGTCAGAAGGACT-3'; and $\alpha$-actin reverse primer, 5'-GAGGCATACAGGGACAACAC-3'. Quantitative PCR was carried out with a 7500 Real Time PCR System (Applied Biosystems, Foster City, CA, USA) using equal amounts of cDNA template. Quantitative PCR analysis of RTP801 or NEDD4 was normalized by $\alpha$-actin and analyzed using the comparative quantification.

\section{Immunofluorescence}

NGF-differentiated PC12 cells and rat primary cortical neurons were fixed with $4 \%$ paraformaldehyde in PBS and stained as previously described [7]. Cortical neurons were permeabilized with PBS containing $0.25 \%$ Triton X-100 during 5 minutes, and blocked with superblock-PBS for 30 minutes. In cell survival assays, transfected viable cells were scored by strip counting [6].

\section{Immunohistochemistry of human sections}

Postmortem human SNpc paraffin-embedded sections from sporadic PD patients and control individuals were obtained from the Neurological Tissue Bank (Biobank-HC-IDIBAPS) and stained as previously reported [6]. Sections were dewaxed with xylene and rehydrated by incubation in ethanol series $(100 \%, 95 \%$, $70 \%$ and $50 \%$ ). Antigen retrieval was performed with TrisEDTA buffer $(10 \mathrm{mM}$ Tris-base $\mathrm{pH}=9,1 \mathrm{mM}$ EDTA, $0.05 \%$ Tween 20 ) in a vegetable steamer at $100^{\circ} \mathrm{C}$ for 20 minutes. Then, slides were blocked in superblock-PBS for 2 hours at room temperature and were incubated with avidin/biotin solution (Vector Laboratories, Burlingame, $\mathrm{CA}$ ). Sections were incubated $\mathrm{O} / \mathrm{N}$ at $4^{\circ} \mathrm{C}$ with antiNEDD4 antibody (Abcam), washed with TBS- $0.025 \%$ Triton X-100 and incubated for 15 minutes with $0.3 \% \mathrm{H}_{2} \mathrm{O}_{2}$ 
in TBS. Secondary antibody incubation was performed for 2 hours at room temperature with biotinylated goat antirabbit secondary antibody (Vector Laboratories). Finally, slides were incubated with ABC Peroxidase Standard Staining Kit (Thermo Fisher Scientific) for 30 minutes and with ImmPACT SG Peroxidase Substrate (Vector Laboratories) for 20 minutes. Sections were washed, dehydrated by incubation in ethanol series $(50 \%, 70 \%$, $95 \%$ and $100 \%$ ) and xylene, mounted with DPX mountant (Sigma-Aldrich) and examined under the microscope.

\section{Statistics}

All experiments were performed at least in triplicate and results are expressed as the mean \pm SEM. Statistical analyses were performed with unpaired Student's $t$ test or when comparing multiple groups with one-way ANOVA with Bonferroni or Newman-Keuls multiple comparison test, as indicated in figure legends. Values of $P<0.05$ were considered as statistically significant.

\section{Abbreviations}

6-OHDA, 6-hydroxydopamine; DSP, dithiobis succinimidyl propionate; eGFP, enhanced green fluorescent protein; HMW, high molecular weight; IB: immunoblot; IP, immunoprecipitation; mTOR, mechanistic target of rapamycin; NEDD4, neural precursor cell expressed developmentally down-regulated protein 4; $\mathrm{NGF}$, nerve growth factor; $\mathrm{NM}+$, neuromelanin positive; PD, Parkinson's disease; shRNA, short hairpin RNA; SPD, sporadic Parkinson's disease; SNpc, substantia nigra pars compacta; UPS, ubiquitin proteasome system; WB, western immunoblotting; WT, wild-type; SNP, single nucleotide polymorphism.

\section{ACKNOWLEDGMENTS}

We thank Dr. Esther Pérez-Navarro, Dr. Hung-En Hsia, and Dr. Sílvia Ginés for helpful discussion. We thank and the staff of the animal facility and DNA core facility (AGCT lab) at the Max Planck Institute of Experimental Medicine. We are indebted to the Neurological Tissue Bank (Biobank-Hospital Clínic-IDIBAPS) and to Dr. Ellen Gelpi, for the human postmortem brain samples and data procurement.

\section{CONFLICTS OF INTERESTS}

The authors declare no conflict of interest.

\section{GRANT SUPPORT}

Supported in part by grants from the Spanish
Ministry of Science and Innovation (SAF2010-21058 and SAF2013-45888R to C.M., SAF2011-29507 to J.A., SAF-2014-57160R to C.M. and J.A.), the PEOPLE Programme - Marie Curie Actions, European Community to C.M. (PIRG08-GA-2010-276957), the Max Planck Society (to H.K.), Fritz Thyssen Foundation (to H.K.), and German Research Foundation (SPP1365/KA3423/11 and KA3423/3-1 to H.K.). Supported also by the crowd funding campaign "SOS recerca en Parkinson" via Goteo. org, Portal d'Avall, S.L. and "Mememtum: early detection of neurological disorders".

\section{Consent for publication}

Informed written consent was received for publication of the manuscript and the patient data presented in Table 1: "Written informed consent was obtained from the patient for publication of their individual details and accompanying images in this manuscript. The consent form is held by the Neurological Tissue Bank (Biobank-Hospital Clínic-IDIBAPS) in the Hospital Clínic (Barcelona) and is available for review by the Editor-inChief.

\section{Ethics statement}

The use of the human SN sections was approved in May $18^{\text {th }} 2015$, by the Bioethics Commission of the University of Barcelona (Secretary Dr. Albert Royes Qui, President: Dr. Jordi Alberch Vié) Institutional Review board: IRB00003099.

\section{REFERENCES}

1. Fahn S. Medical treatment of Parkinson's disease. J Neurol. 1998; 245: P15-24.

2. Dauer W, Przedborski S. Parkinson's disease: mechanisms and models. Neuron. 2003; 39: 889-909.

3. Marras C, Lang A. Invited article: changing concepts in Parkinson disease: moving beyond the decade of the brain. Neurology. 2008; 70: 1996-2003.

4. Levy OA, Malagelada C, Greene LA. Cell death pathways in Parkinson's disease: Proximal triggers, distal effectors, and final steps. Apoptosis. 2009; 14: 478-500.

5. Shoshani T, Faerman A, Mett I, Zelin E, Tenne T, Gorodin S, Moshel Y, Elbaz S, Budanov A, Chajut A, Kalinski H, Kamer I, Rozen A, et al. Identification of a novel hypoxiainducible factor 1-responsive gene, RTP801, involved in apoptosis. Mol Cell Biol. 2002; 22: 2283-93.

6. Malagelada C, Ryu EJ, Biswas SC, Jackson-Lewis V, Greene LA. RTP801 is elevated in Parkinson brain substantia nigral neurons and mediates death in cellular models of Parkinson's disease by a mechanism involving mammalian target of rapamycin inactivation. J Neurosci. 
2006; 26: 9996-10005.

7. Malagelada C, Jin ZH, Greene LA. RTP801 is induced in Parkinson's disease and mediates neuron death by inhibiting Akt phosphorylation/activation. J Neurosci. 2008; 28: 14363-71.

8. Romani-Aumedes J, Canal M, Martin-Flores N, Sun X, Perez-Fernandez V, Wewering S, Fernandez-Santiago R, Ezquerra M, Pont-Sunyer C, Lafuente A, Alberch J, Luebbert H, Tolosa E, et al. Parkin loss of function contributes to RTP801 elevation and neurodegeneration in Parkinson's disease. Cell Death Dis. 2014; 5: e1364.

9. Kimball SR, Do AN, Kutzler L, Cavener DR, Jefferson LS. Rapid turnover of the mTOR complex 1 (mTORC1) repressor REDD1 and activation of $\mathrm{mTORC} 1$ signaling following inhibition of protein synthesis. J Biol Chem. 2008; 283: 3465-75.

10. Malagelada C, Jin ZH, Jackson-Lewis V, Przedborski S, Greene LA. Rapamycin protects against neuron death in in vitro and in vivo models of Parkinson's disease. J Neurosci. 2010; 30: 1166-75.

11. Boase NA, Kumar S. NEDD4: The founding member of a family of ubiquitin-protein ligases. Gene. 2015; 557:11322.

12. Wang X, Trotman LC, Koppie T, Alimonti A, Chen Z, Gao Z, Wang J, Erdjument-Bromage H, Tempst P, CordonCardo C, Pandolfi PP, Jiang X. NEDD4-1 Is a ProtoOncogenic Ubiquitin Ligase for PTEN. Cell. 2007; 128: 129-39.

13. Kawabe H, Neeb A, Dimova K, Young SM, Takeda M, Katsurabayashi S, Mitkovski M, Malakhova OA, Zhang DE, Umikawa M, Kariya K ichi, Goebbels S, Nave KA, et al. Regulation of Rap2A by the Ubiquitin Ligase Nedd4-1 Controls Neurite Development. Neuron. 2010; 65: 358-72.

14. Drinjakovic J, Jung H, Campbell DS, Strochlic L, Dwivedy A, Holt CE. E3 Ligase Nedd4 Promotes Axon Branching by Downregulating PTEN. Neuron. 2010; 65: 341-57.

15. Tofaris GK, Kim HT, Hourez R, Jung J-W, Kim KP, Goldberg a. L. Ubiquitin ligase Nedd4 promotes -synuclein degradation by the endosomal-lysosomal pathway. Proc Natl Acad Sci. 2011; 108: 17004-9.

16. Davies SE, Hallett PJ, Moens T, Smith G, Mangano E, Kim HT, Goldberg AL, Liu JL, Isacson O, Tofaris GK. Enhanced ubiquitin-dependent degradation by Nedd4 protects against ??-synuclein accumulation and toxicity in animal models of Parkinson's disease. Neurobiol Dis. 2014; 64: 79-87.

17. Srinivasan BS, Doostzadeh J, Absalan F, Mohandessi S, Jalili R, Bigdeli S, Wang J, Mahadevan J, Lee CLG, Davis RW, Langston JW, Ronaghi M. Whole genome survey of coding SNPs reveals a reproducible pathway determinant of Parkinson disease. Hum Mutat. 2009; 30: 228-38.

18. Greene LA, Tischler AS. Establishment of a noradrenergic clonal line of rat adrenal pheochromocytoma cells which respond to nerve growth factor. Proc Natl Acad Sci U S A.
1976; 73: 2424-8.

19. Sun Liu, J., Crary, J.F., Malagelada, C., Sulzer, D., Greene, L.A., Levy, O.A. X. ATF4 protects against neuronal death in cellular PD models by maintaining levels of parkin. $\mathrm{J}$ Neurosci. 2013; 33:2398-407.

20. Rideout H, Stefanis L. Proteasomal Inhibition-Induced Inclusion Formation and Death in Cortical Neurons Require Transcription and Ubiquitination. Mol Cell Neurosci. 2002; 21: 223-38.

21. Wibo M, Poole B. Protein degradation in cultured cells. II. The uptake of chloroquine by rat fibroblasts and the inhibition of cellular protein degradation and cathepsin B1. J Cell Biol. 1974; 63: 430-40.

22. Suzuki T, Nakagawa M, Yoshikawa A, Sasagawa N, Yoshimori T, Ohsumi Y, Nishino I, Ishiura S, Nonaka I. The first molecular evidence that autophagy relates rimmed vacuole formation in chloroquine myopathy. $\mathrm{J}$ Biochem. 2002; 131: 647-51.

23. Hicke L, Dunn R. Regulation of membrane protein transport by ubiquitin and ubiquitin-binding proteins. Annu Rev Cell Dev Biol. 2003; 19: 141-72.

24. Gorski J a, Talley T, Qiu M, Puelles L, Rubenstein JLR, Jones KR. Cortical excitatory neurons and glia, but not GABAergic neurons, are produced in the Emx1-expressing lineage. J Neurosci. 2002; 22: 6309-14.

25. Gorski JA, Zeiler SR, Tamowski S, Jones KR. Brainderived neurotrophic factor is required for the maintenance of cortical dendrites. J Neurosci. 2003; 23: 6856-65.

26. Parkkinen L, O'Sullivan SS, Collins C, Petrie A, Holton JL, Revesz T, Lees AJ. Disentangling the Relationship between Lewy bodies and nigral neuronal loss in Parkinson's disease. J Parkinsons Dis. 2011; 1: 277-86.

27. Molitoris JK, McColl KS, Swerdlow S, Matsuyama M, Lam M, Finkel TH, Matsuyama S, Distelhorst CW. Glucocorticoid elevation of dexamethasone-induced gene 2 (Dig2/RTP801/REDD1) protein mediates autophagy in lymphocytes. J Biol Chem. 2011; 286: 30181-9.

28. Sancak Y, Bar-Peled L, Zoncu R, Markhard AL, Nada S, Sabatini DM. Ragulator-rag complex targets mTORC1 to the lysosomal surface and is necessary for its activation by amino acids. Cell. 2010; 141: 290-303.

29. McNaught KS, Olanow CW, Halliwell B, Isacson O, Jenner P. Failure of the ubiquitin-proteasome system in Parkinson's disease. Nat Rev Neurosci. 2001; 2: 589-94.

30. Pan T, Kondo S, Le W, Jankovic J. The role of autophagylysosome pathway in neurodegeneration associated with Parkinson's disease. Brain. 2008; 131: 1969-78.

31. Nathan J a, Kim HT, Ting L, Gygi SP, Goldberg AL. Why do cellular proteins linked to K63-polyubiquitin chains not associate with proteasomes? EMBO J. 2013; 32: 552-65.

32. Rotin D, Kumar S. Physiological functions of the HECT family of ubiquitin ligases. Nat Rev Mol Cell Biol. 2009; 10: 398-409. 
33. Lu PJ, Zhou XZ, Shen M, Lu KP. Function of WW domains as phosphoserine- or phosphothreonine-binding modules. Science. 1999; 283: 1325-8.

34. Katiyar S, Liu E, Knutzen CA, Lang ES, Lombardo CR, Sankar S, Toth JI, Petroski MD, Ronai Z, Chiang GG. REDD1, an inhibitor of mTOR signalling, is regulated by the CUL4A-DDB1 ubiquitin ligase. EMBO Rep. 2009; 10: 866-72.

35. Zhao Y, Xiong X, Jia L, Sun Y. Targeting Cullin-RING ligases by MLN4924 induces autophagy via modulating the HIF1-REDD1-TSC1-mTORC1-DEPTOR axis. Cell Death Dis. 2012; 3: e386.

36. Kitada T, Asakawa S, Hattori N, Matsumine H, Yamamura Y, Minoshima S, Yokochi M, Mizuno Y, Shimizu N. Mutations in the parkin gene cause autosomal recessive juvenile parkinsonism. Nature.1998; 392: 605-8.

37. Shimura H, Hattori N, Kubo S, Mizuno Y, Asakawa S, Minoshima S, Shimizu N, Iwai K, Chiba T, Tanaka K, Suzuki T. Familial Parkinson disease gene product, parkin, is a ubiquitin-protein ligase. Nat Genet. 2000; 25: 302-5.

38. Chung KK, Thomas B, Li X, Pletnikova O, Troncoso JC, Marsh L, Dawson VL, Dawson TM. S-nitrosylation of parkin regulates ubiquitination and compromises parkin's protective function. Science. 2004; 304: 1328-31.

39. Winklhofer KF, Henn IH, Kay-Jackson PC, Heller U, Tatzelt J. Inactivation of parkin by oxidative stress and C-terminal truncations: a protective role of molecular chaperones. J Biol Chem. 2003; 278: 47199-208.
40. LaVoie MJ, Ostaszewski BL, Weihofen A, Schlossmacher MG, Selkoe DJ. Dopamine covalently modifies and functionally inactivates parkin. Nat Med.2005; 11: 1214 21.

41. Fan CD, Lum MA, Xu C, Black JD, Wang X. Ubiquitindependent regulation of phospho-AKT Dynamics by the ubiquitin E3 LIGASE, NEDD4-1, in the insulin-like growth factor-1 response. J Biol Chem. 2013; 288: 1674-84.

42. Vecchione A, Marchese A, Henry P, Rotin D, Morrione A. The Grb10/Nedd4 complex regulates ligand-induced ubiquitination and stability of the insulin-like growth factor I receptor. Mol Cell Biol. 2003; 23: 3363-72.

43. Yu Y, Yoon S-O, Poulogiannis G, Yang Q, Ma XM, Villén J, Kubica N, Hoffman GR, Cantley LC, Gygi SP, Blenis J. Phosphoproteomic analysis identifies Grb10 as an mTORC1 substrate that negatively regulates insulin signaling. Science. 2011; 332: 1322-6.

44. Hsia H-E, Kumar R, Luca R, Takeda M, Courchet J, Nakashima J, Wu S, Goebbels S, An W, Eickholt BJ, Polleux F, Rotin D, Wu H, et al. Ubiquitin E3 ligase Nedd41 acts as a downstream target of PI3K/PTEN-mTORC1 signaling to promote neurite growth. Proc Natl Acad Sci U S A. 2014; 111: 13205-10. 